\title{
Controlled Decompression Attenuates Compressive Injury following Traumatic Brain Injury via TREK-1-Mediated Inhibition of Necroptosis and Neuroinflammation
}

\author{
Tao Chen $(\mathbb{D}$, Xiao Qian, Jie Zhu, Li-Kun Yang $\mathbb{D}$, and Yu-Hai Wang $\mathbb{D}$ \\ Department of Neurosurgery, The 904th Hospital of PLA, Medical School of Anhui Medical University, Wuxi, \\ Jiangsu 214044, China \\ Correspondence should be addressed to Li-Kun Yang; yanglikun904@163.com and Yu-Hai Wang; prof_wyh101@163.com
}

Received 12 August 2021; Accepted 24 September 2021; Published 8 November 2021

Academic Editor: Yanqing Wu

Copyright (C) 2021 Tao Chen et al. This is an open access article distributed under the Creative Commons Attribution License, which permits unrestricted use, distribution, and reproduction in any medium, provided the original work is properly cited.

\begin{abstract}
Decompressive craniectomy is an effective strategy to reduce intracranial hypertension after traumatic brain injury (TBI), but it is related to many postoperative complications, such as delayed intracranial hematoma and diffuse brain swelling. Our previous studies have demonstrated that controlled decompression (CDC) surgery attenuates brain injury and reduces the rate of complications after TBI. Here, we investigated the potential molecular mechanisms of CDC in experimental models. The in vitro experiments were performed in a traumatic neuronal injury (TNI) model following compression treatment in primary cultured cortical neurons. We found that compression aggravates TNI-induced neuronal injury, which was significantly attenuated by CDC for $2 \mathrm{~h}$ or $3 \mathrm{~h}$. The results of immunocytochemistry showed that CDC reduced neuronal necroptosis and activation of RIP3 induced by TNI and compression, with no effect on RIP1 activity. These protective effects were associated with decreased levels of inflammatory cytokines and preserved intracellular $\mathrm{Ca}^{2+}$ homeostasis. In addition, the expression of the two-pore domain $\mathrm{K}^{+}$channel TREK-1 and its activity was increased by compression and prolonged by CDC. Treatment with the TREK-1 blockers, spadin or SID1900, could partially prevent the effects of CDC on intracellular $\mathrm{Ca}^{2+} \mathrm{metabolism}^{2}$ necroptosis, and neuronal injury following TNI and compression. Using a traumatic intracranial hypertension model in rats, we found that $\mathrm{CDC}$ for $20 \mathrm{~min}$ or $30 \mathrm{~min}$ was effective in alleviating brain edema and locomotor impairment in vivo. CDC significantly inhibited neuronal necroptosis and neuroinflammation and increased TREK-1 activation, and the CDC-induced protection in vivo was attenuated by spadin and SID1900. In summary, CDC is effective in alleviating compressive neuronal injury both in vitro and in vivo, which is associated with the TREK-1-mediated attenuation of intracellular Ca ${ }^{2+}$ overload, neuronal necroptosis, and neuroinflammation.
\end{abstract}

\section{Introduction}

Traumatic brain injury (TBI) has been considered as one of the most complex human diseases because of the complexity of brain damage mechanisms and poor prognosis. For patients with uncontrollable high intracranial pressure, decompressive craniectomy is an effective strategy to reduce traumatic intracranial hypertension and improve prognosis [1]. However, the standard procedure of rapid decompression is associated with many postoperative complications, such as delayed intracranial hematoma and diffuse brain swelling $[2,3]$. Our previous studies have demonstrated that the controlled decompression (CDC) surgery attenuates brain injury and reduces the rates of complications after TBI [4-6]. However, the underlying molecular mechanism has not been determined.

In the central nervous system (CNS), a variety of cellular physiological processes, including neurotransmitter release, neuronal excitability, and plasticity, are regulated by ion channels, especially $\mathrm{Ca}^{2+}$ and $\mathrm{K}^{+}$ions [7]. The tandem of pore domain in weak inwardly rectifying $\mathrm{K}^{+}$channel- (TWIK-) related $\mathrm{K}^{+}$channels (TREK) belongs to the recently discovered two-pore domain $\mathrm{K}^{+}\left(\mathrm{K}_{2 \mathrm{P}}\right)$ channels, which include 15 members grouped in six subfamilies and are responsible for 
maintaining the neuronal resting membrane potential [8]. TREK-1, also known as KCNK2 or $\mathrm{K}_{2 \mathrm{P}} 2.1$, is highly expressed in the lung and brain, from the prefrontal cortex, hippocampus, and midbrain to the spinal cord [9]. Since its cloning two decades ago, the physiological importance of TREK-1 is constantly being discovered. TREK-1 is located at both presynaptic and postsynaptic components, which are its key roles in maintaining the resting membrane potential and neurotransmitter release. Its activation is regulated by various physical and chemical stimuli, such as mechanical stretch, Gq-coupled group I mGluRs, and Gs-coupled 5-HR4 receptor serotonin [10]. Increasing evidence has been demonstrated that dysfunction of TREK-1 channels is involved in multiple neurological pathologies, including depression, pain, epilepsy, and ischemia [11]. In TREK-1 gene knockout mice, TREK-1 null was found to increase neuronal excitability and enhance excitatory and inhibitory postsynaptic currents, thereby impairing cognitive function [12]. In addition, TREK-1 was found to be upregulated by ischemia in astrocytes to enhance glutamate clearance and block neuronal death [13]. However, the role of TREK-1 in traumatic intracranial hypertension conditions has not been fully determined.

In the current study, we investigated the effect of CDC on compressive injury following neuronal trauma in an in vitro model using primary cultured cortical neurons and also in an in vivo traumatic intracranial hypertension model in rats. Considering the effect of mechanical force on TREK1 activation, we assessed the potential role of TREK-1 in $\mathrm{CDC}$-induced protection.

\section{Materials and Methods}

2.1. Primary Culture of Cortical Neurons. Because neurons in the cerebral cortex are most vulnerable under traumatic intracranial hypertension, primary cultured cortical neurons were obtained from SD rats to establish the experimental models as previously described [14]. All the animal research procedures were approved by the Anhui Medical University Committee of Animal Research.

2.2. In Vitro Model and Treatments. The TNI model was performed according to our previously published method [15]. In brief, traumatic injury was performed on cultured neurons by using a rotating scribe injury device, which consisted of a rotating cylinder with ten holes, steel needles, and a permanent magnet. The cylinder holes are distributed at the same interval from the center, and these holes allowed the ten steel needles to freely cross through. A magnet is placed under the culture dish, which ensured that the steel needles could cling to the cell layer as the cylinder rotated. After one turn of this device, ten concentric circular scratches were produced in the neuronal layer with equal distances $(1.5 \mathrm{~mm})$ between the scratches. After TNI, neurons were exposed to continuous high pressure of $0.5 \mathrm{MPa}$ according to our previous published paper [16]. Briefly, a custom-made compression apparatus in vitro was applied to expose neurons to continuous high pressure for $3 \mathrm{~h}$. The TREK-1 blockers, spadin and SID1900, were obtained from the Mycell Biotech Company (Shanghai, China) and diluted in neurobasal medium to achieve the final concentration of $1 \mu \mathrm{M}$ or $30 \mu \mathrm{M}$, respectively.

2.3. Hematoxylin-Eosin (HßE) Staining. At $12 \mathrm{~h}$ after TNI and compression treatment, cortical neurons were washed with phosphate buffer saline (PBS) and fixed with $4 \%$ paraformaldehyde for $30 \mathrm{~min}$. Neurons were deparaffinized with xylene, stained by $\mathrm{H} \& \mathrm{E}$, and then assessed by a pathologist for neuronal loss under an Olympus U-DO3 light microscope (Tokyo, Japan).

2.4. LDH Release. The neurotoxicity in cortical neurons was determined by measuring LDH release at $12 \mathrm{~h}$ after TNI and compression using a kit according to the manufacturer's protocol (Jiancheng Bioengineering Institute, Nanjing, Jiangsu, China).

2.5. Cell Viability Assay. Cell viability of cortical neurons was determined by calcein AM assay at $12 \mathrm{~h}$ after TNI and compression using a kit according to the manufacturer's protocol (Enzo Life Sciences, Farmingdale, NY, USA).

2.6. Immunocytochemistry. For immunocytochemistry, the neurons were cultured in poly-D-lysine-coated coverslips and treated with TNI, compression, or CDC. After being fixed with $4 \%$ paraformaldehyde, permeabilized with $0.1 \%$ Triton X-100, and washed with PBS for three times, neurons were blocked by $5 \%$ bovine serum albumin. Incubation with the RIP1 (1:100, \#3493, Cell Signaling), RIP3 (1:200, ab62344, Abcam), and TREK-1 (1:50, sc-398449, Santa Cruz) primary antibodies was performed at $4^{\circ} \mathrm{C}$ overnight. After being washed by PBS with Tween-20 (PBST) for three times, the samples were incubated with the secondary antibody at $37^{\circ} \mathrm{C}$ for $1 \mathrm{~h}$. Then, incubation with $4^{\prime}, 6$-diamidino-2-phenylindole (DAPI) was performed to stain the nuclei, and the images were obtained using a Leica SP5 II confocal microscope.

2.7. Enzyme-Linked Immunosorbent Assay (ELISA). The levels of inflammatory cytokines, including TNF- $\alpha$, IL- $1 \beta$, IL-6, INF- $\gamma$, and IL-18, were measured by ELISA kits following the manufacturer's protocols (Anoric-Bio, Tianjin, China).

2.8. $\mathrm{Ca}^{2+}$ Imaging. $\mathrm{Ca}^{2+}$ imaging was performed using the $\mathrm{Ca}^{2+}$ indicator Fura-2 AM to measure the intracellular $\mathrm{Ca}^{2+}$ concentrations [17]. The neurons cultured in coverslips were loaded with $5 \mu \mathrm{M}$ Fura-2 AM in HBSS solution for $30 \mathrm{~min}$ and equilibrated lucifugally for $30 \mathrm{~min}$. Cells were excited at 345 and $385 \mathrm{~nm}$ using a confocal laser scanning microscope, and the emission fluorescence at $510 \mathrm{~nm}$ was recorded. The fluorescence values were then plotted against time and shown as $F / F_{0}$.

2.9. Patch Clamp Electrophysiology. The single TREK-1 channel currents were recorded in cortical neurons using the inside-out patch clamp configuration, and the bath solution contained the following: $130 \mathrm{mM} \mathrm{KCl}, 10 \mathrm{x}$ HEPES, $1 \mathrm{mM}$ $\mathrm{MgCl}_{2}, 5 \mathrm{mM}$ EGTA, and $10 \mu \mathrm{M} \mathrm{Ca}^{2+}$. The experiments were 

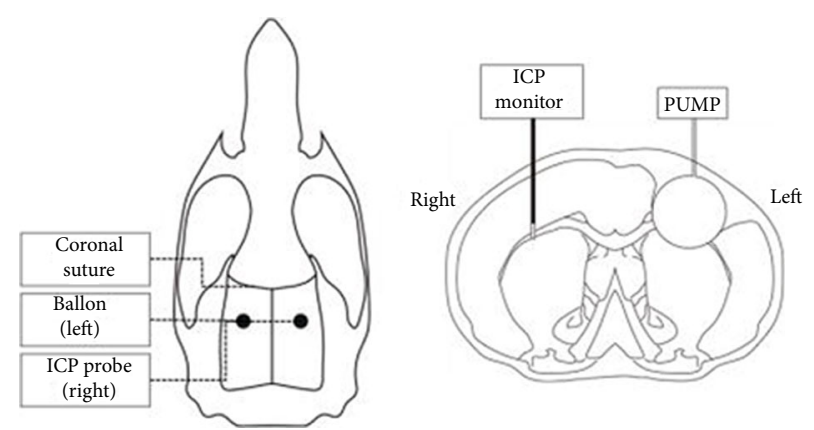

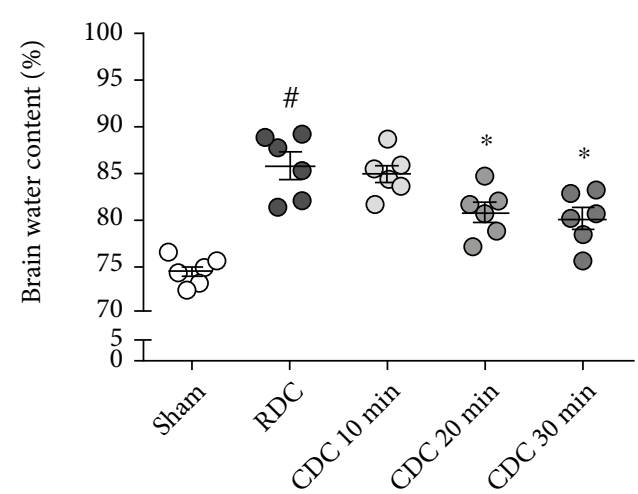

(b)

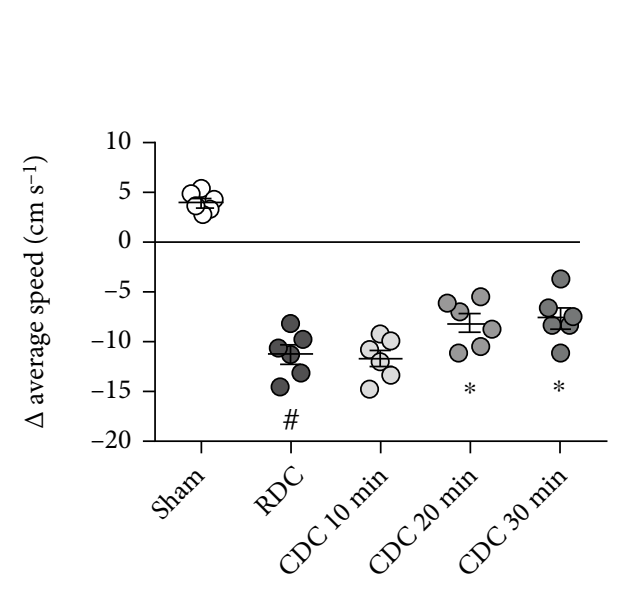

(c)
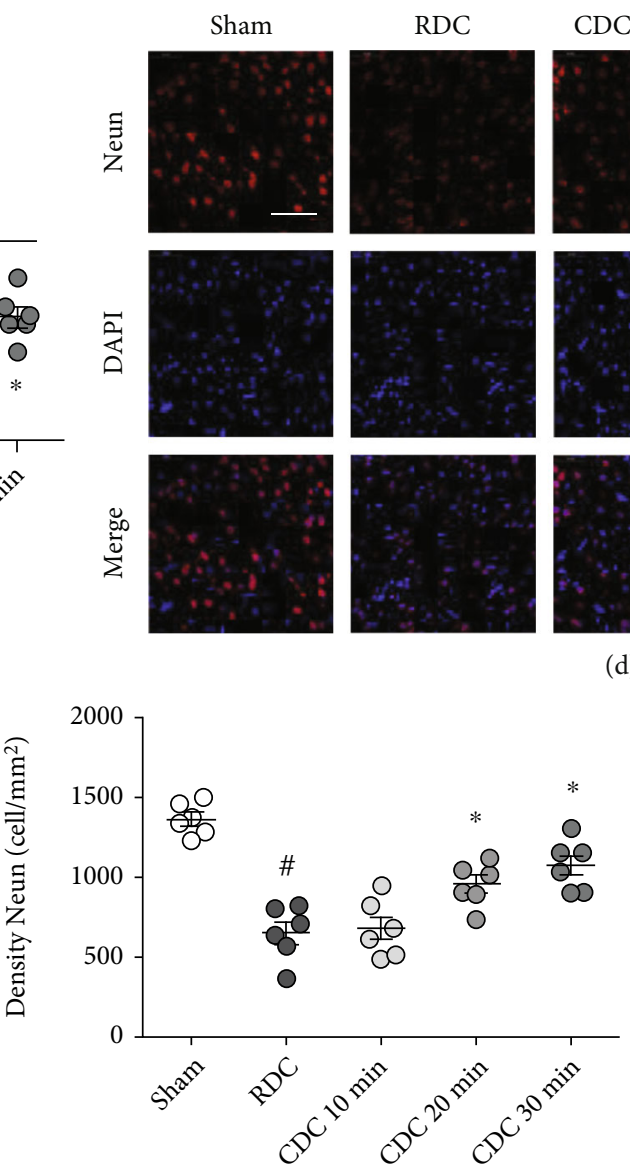

RDC
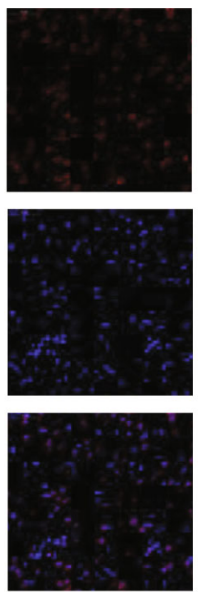

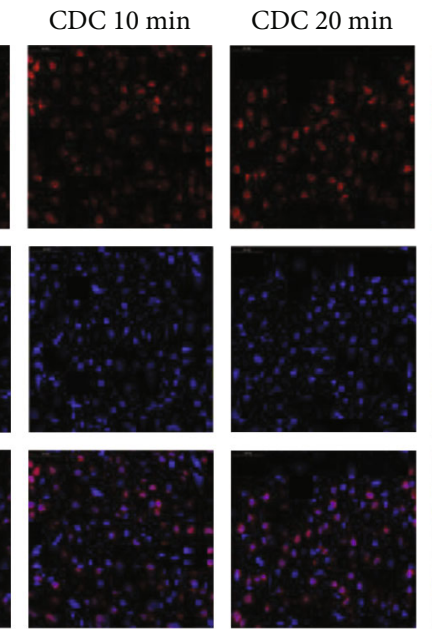

(d)

(e)

FIGURE 1: CDC attenuates brain damage after traumatic intracranial hypertension. (a) The horizontal and coronal schematic diagrams of the traumatic intracranial hypertension model. (b) Brain water content assay shows that CDC for 20 min or 30 min reduced brain edema compared to RDC. (c) The neurological assay shows that CDC for $20 \mathrm{~min}$ or $30 \mathrm{~min}$ attenuates locomotor impairment compared to RDC. (d, e) Typical pictures of NeuN staining (d) and quantification (e) show that CDC for 20 min or 30 min decreases neuronal loss compared to RDC. The data was represented as means \pm SEM. ${ }^{*} p<0.05$ vs. sham group and ${ }^{*} p<0.05$ vs. RDC group.

performed at membrane voltages of $-40 \mathrm{mV}$. TREK- 1 currents were filtered at $1 \mathrm{kHz}$ and digitized at $5 \mathrm{kHz}$. Analysis was performed using Clampfit 9.2 (MDS Analytical Technologies).

2.10. Traumatic Intracranial Hypertension Model. Traumatic intracranial hypertension was induced using a balloon compression method in SD rats. Briefly, anesthesia was induced via 5\% isoflurane (RWD, Guangdong, China) with a $1: 1$
$\mathrm{N}_{2} \mathrm{O} / \mathrm{O}_{2}$ mixture and was then maintained with a $2.5 \%$ isoflurane with $1: 1 \mathrm{~N}_{2} \mathrm{O} / \mathrm{O}_{2}$ mixture by a face mask. The rats were placed on a fixing frame in the prone position. $2 \%$ lidocaine was injected subcutaneously near the midline of the skull to minimize the influence of skin incision on blood pressure. The fur around the midline of the skull was cleared with an electronic clipper and the skin of the rat was disinfected by using $75 \%$ alcohol. We incised the scalp along 

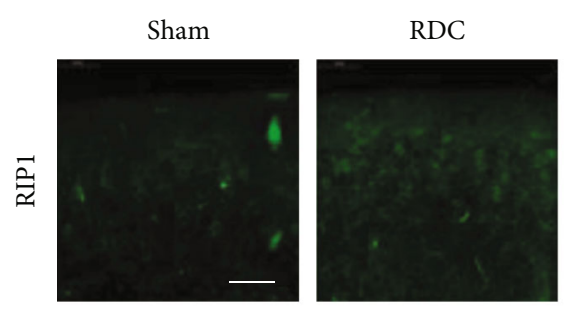

CDC
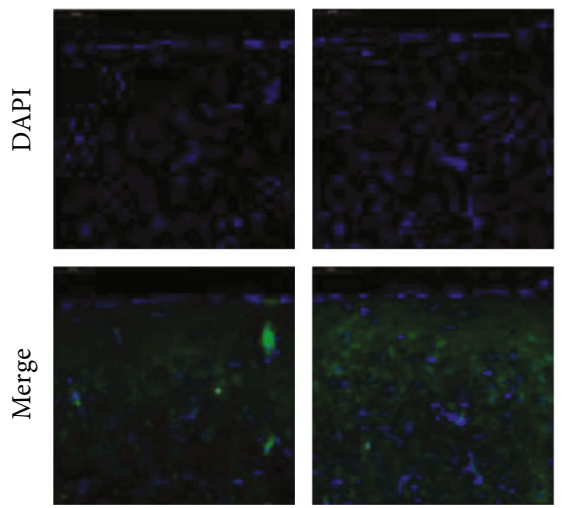

(a)
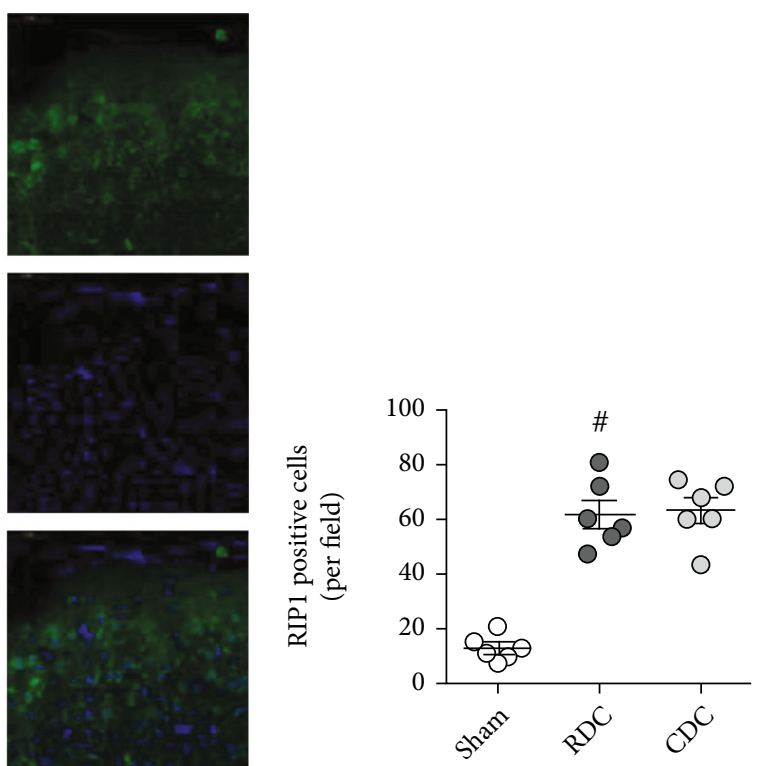

(b)
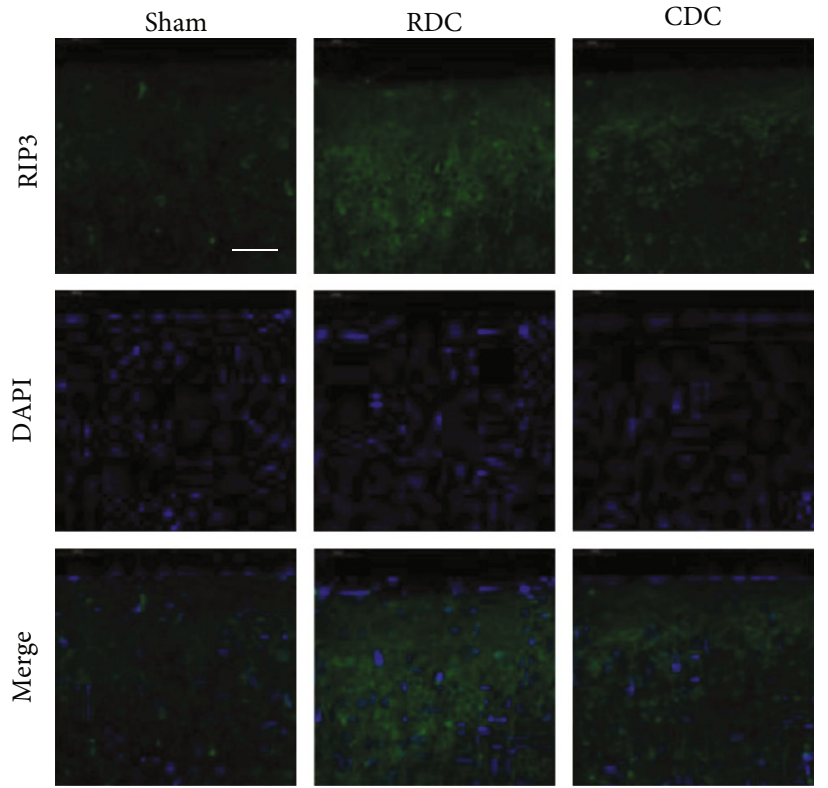

(c)

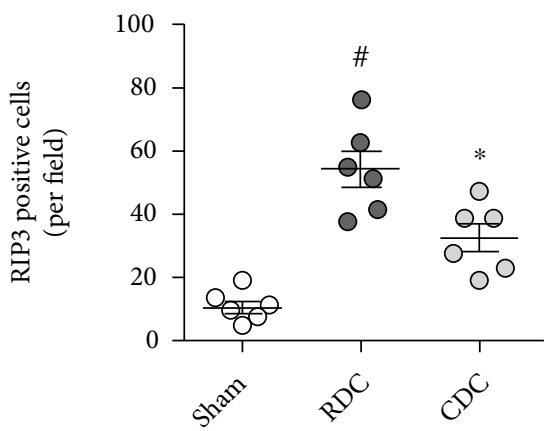

(d)

Figure 2: Continued. 

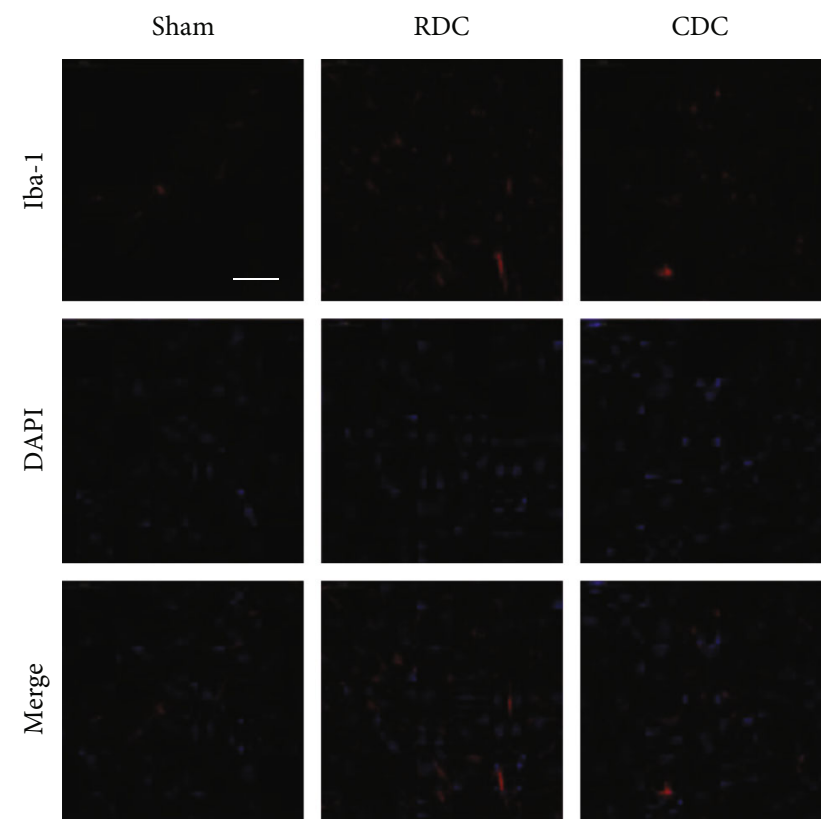

(e)

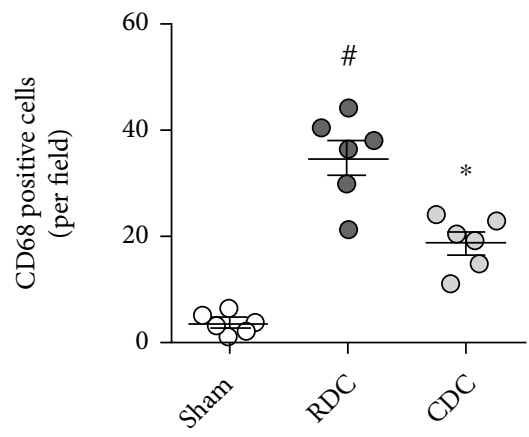

(f)

FIGURE 2: CDC alleviates neuronal necroptosis and neuroinflammation. (a, b) Typical pictures of RIP1 staining (a) and quantification (b) show that CDC had no effect on the RDC-induced increase of RIP1 expression. The scale bar is $50 \mu \mathrm{m}$. (c, d) Typical pictures of RIP3 staining (c) and quantification (d) show that CDC attenuates the RIP3 expression compared to RDC. The scale bar is $50 \mu \mathrm{m}$. (e, f) Typical pictures of CD68 staining (e) and quantification (f) show that CDC attenuates microglial activation compared to RDC. The scale bar is $50 \mu \mathrm{m}$. The data was represented as means \pm SEM. ${ }^{*} p<0.05$ vs. sham group and ${ }^{*} p<0.05$ vs. RDC group.

the midline for $2.5 \mathrm{~cm}$, separated the subcutaneous tissue, and striped the periosteum to expose the anterior- and posterior-sagittal suture adequately. We made two holes using a dental drill (JSDA, JD700, China) bilaterally in the position of $0.5 \mathrm{~cm}$ by the side of the midline and $0.6 \mathrm{~cm}$ behind the anterior-sagittal suture and carefully removed the skull fragment to expose the cerebral dura mater without inflicting additional damage. The balloon system is composed of an embolization balloon (Balt 2, Montmorency, France) mounted on an intervention catheter (MAGIC, Montmorency, France). The balloon was inserted into the left hole carefully towards the frontotemporal side, and the dura mater was kept intact during the insertion so that the balloon would only lead to epidural compression. The catheter was connected to a pressure pump (Merit Basix Touch Inflation Device, IN4130, USA) to control the amount of injection fluid accurately. The ICP transducer was inserted into the brain at the depth of around $0.5 \mathrm{~cm}$ and was connected to an ICP monitoring device (Codman, Johnson and Johnson Medical, 82-6635, USA). After the insertions of the ICP probe and balloon, the bone holes were sealed using dental cement (Dentsply, Jeltrate Alginate Impression Material, USA) (Figure 1(a)). When the balloon was inflated by the pressure pump gradually, the variation trend of ICP and CPP was recorded.

\subsection{In Vivo Experimental Design. Experiment 1 (Figure 1):} the animals were randomly assigned into five groups: the sham group, rapid decompression group (RDC), controlled decompression for $10 \mathrm{~min}$ group (CDC $10 \mathrm{~min}$ ), controlled decompression for $20 \mathrm{~min}$ group (CDC $20 \mathrm{~min}$ ), and con- trolled decompression for $30 \mathrm{~min}$ group (CDC $30 \mathrm{~min}$ ). The surgery of the sham group merely included skin incision, skull exposure, bone hole drilling, and the insertion of the ICP probe and balloon for $30 \mathrm{~min}$ without inflation. For the other four groups, we inflated the balloon with a pump until the value of ICP reached to the level of $30 \mathrm{mmHg}$ which was maintained for $30 \mathrm{~min}$. As for the RDC group, normal saline (NS) was pumped out rapidly within 3 seconds to reduce ICP suddenly after $30 \mathrm{~min}$ ballooninflation. On the contrary, the ICP was lowered gradually in the CDC group, and the whole process lasted for $10 \mathrm{~min}, 20 \mathrm{~min}$, or $30 \mathrm{~min}$.

Experiment 2 (Figures 2 and 3(a) and 3(b)): the animals were randomly assigned into three groups: Sham group, RDC group and CDC group. The rats in Sham and RDC group were treated as mentioned in experiment 1 . The animals in CDC group were treated with CDC procedure for $30 \mathrm{~min}$ as mentioned in experiment 1 .

Experiment 3 (Figures 3(c) and 3(d)): the animals were randomly assigned into five groups: the sham group, RDC group, CDC group, CDC and spadin group (CDC+spadin), and CDC and SID1900 group (CDC+SID1900). The rats in the first three groups were treated as experiment 2. For the other two groups, rats were pretreated with spadin or SID1900 via the left cerebral ventricle (coordinates relative to bregma: $0.1 \mathrm{~mm}$ posterior, $1 \mathrm{~mm}$ lateral, and $2 \mathrm{~mm}$ deep) before the surgery.

2.12. Measurement of Brain Edema. Brian edema was determined by measuring brain water content using the standard wet and dry method [18]. 


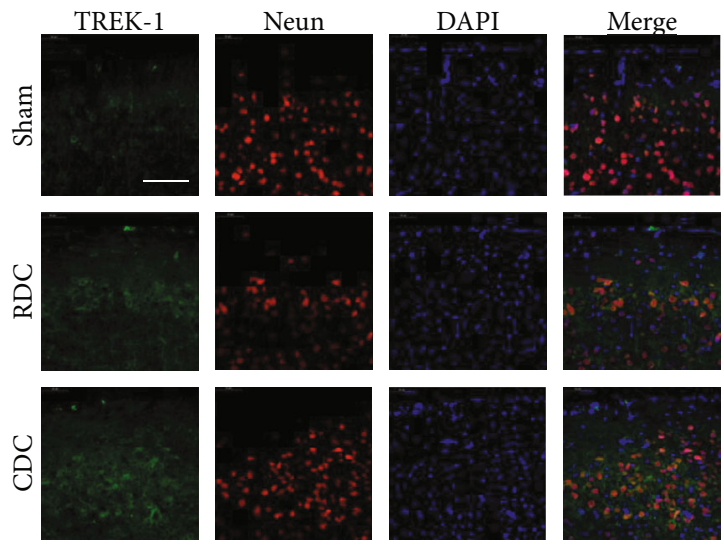

(a)

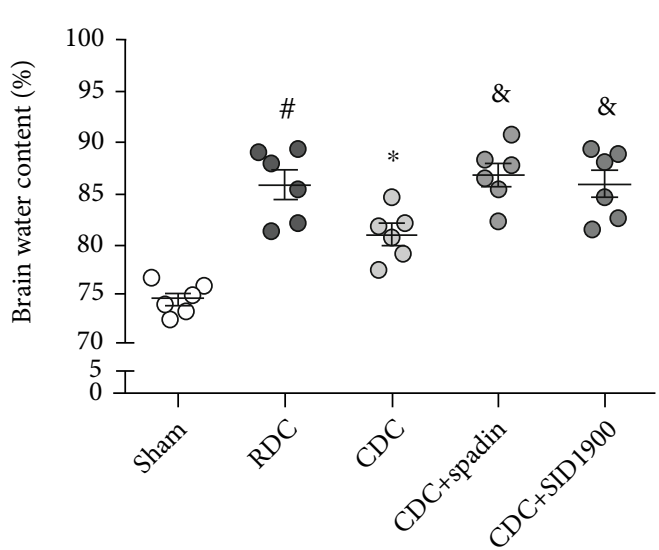

(c)

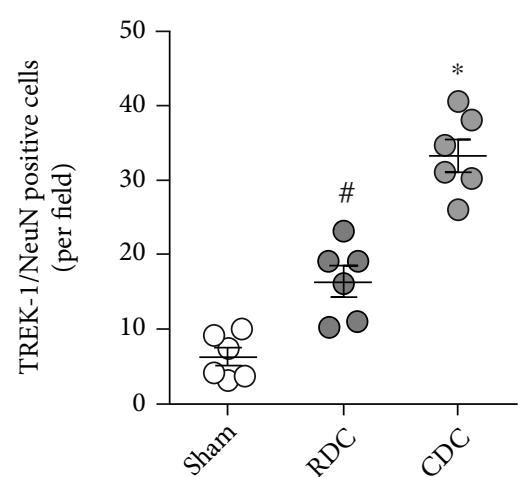

(b)

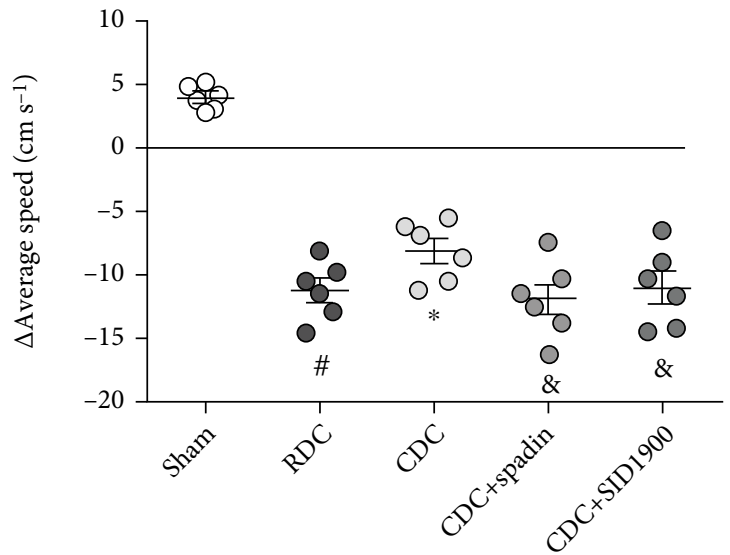

(d)

FIGURE 3: CDC exerts neuroprotective effects via activating TREK-1 in vivo. (a, b) Typical pictures of TREK-1 staining (a) and quantification (b) show that CDC further increased the expression of TREK-1 in cortical neurons compared to RDC. The scale bar is $50 \mu \mathrm{m}$. (c) Brain water content assay shows that the CDC-induced inhibition of brain edema was prevented by spadin and SID1900. (d) The neurological assay shows that the CDC-induced preservation of locomotor function was reversed by spadin and SID1900. The data was represented as means \pm SEM. ${ }^{*} p<0.05$ vs. sham group, ${ }^{*} p<0.05$ vs. RDC group, and ${ }^{*} p<0.05$ vs. CDC group.

2.13. Neurological Function Assay. The CatWalk XT automated gait analysis system (Noldus Information Technology, Wageningen, the Netherlands) was used to measure the locomotor function as previously described [19].

2.14. Immunostaining in Brain Sections. The sections were washed three times with PBST for $5 \mathrm{~min}$ and were blocked by $10 \%$ normal bovine serum (Gibco, United States) in PBST for $1 \mathrm{~h}$ at room temperature. After that, the sections were incubated at $4{ }^{\circ} \mathrm{C}$ overnight in $5 \%$ normal goat serum (NGS) in PBST with the following primary antibodies: NeuN (1:200, \#24307, Cell Signaling), RIP1 (1:100, \#3493, Cell Signaling), RIP3 (1:200, ab62344, Abcam), CD68 (1:200, ab125212, Abcam), and TREK-1 (1:50, sc-398449, Santa Cruz). After being washed for three times by PBST, the sections were incubated with secondary antibodies conjugated to Alexa Fluor (1:800, Invitrogen, United States) for $1 \mathrm{~h}$ at $37^{\circ} \mathrm{C}$. DAPI was administrated to counterstain the nuclei, and the images were obtained using a Leica SP5 II confocal microscope.
2.15. Statistical Analysis. Data represent the mean and standard error of the mean (SEM). Student's $t$ test and repeated measure analysis of variance with Student-Newman-Keuls post hoc test were performed for all statistical significance analyses using GraphPad Prism 6.0 software. All experiments were repeated at least for three times.

\section{Results}

3.1. Compression Aggravates Neuronal Injury after TNI in Cortical Neurons. To mimic intracranial hypertension after TBI in vitro, primary cultured cortical neurons were treated with traumatic injury and compression of $0.5 \mathrm{MPa}$ pressure for $3 \mathrm{~h}$. The results of H\&E staining showed that the neuronal loss induced by TNI was significantly increased by compression (Figure 4(a)). LDH release was measured to determine cytotoxicity, and the TNI-induced increase in $\mathrm{LDH}$ release in cortical neurons was enhanced by compression (Figure 4(b)). In addition, the neuronal viability was assayed by measuring the calcein AM signaling (Figure 4(c)). 


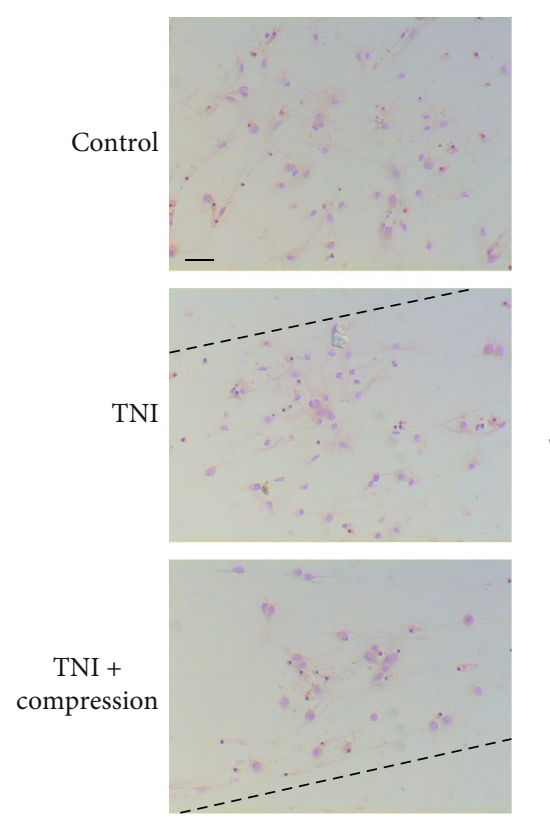

(a)

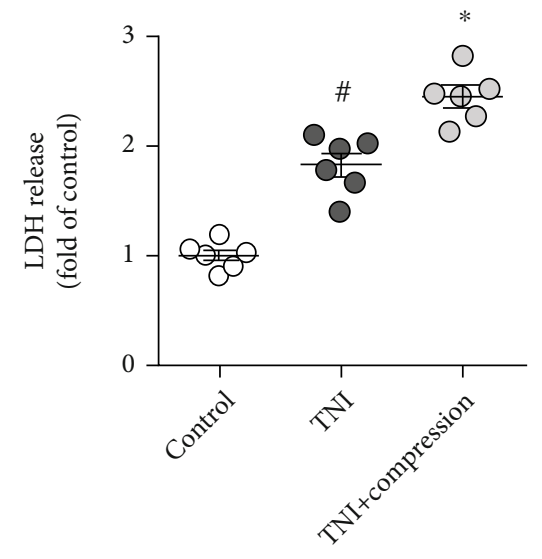

(b)

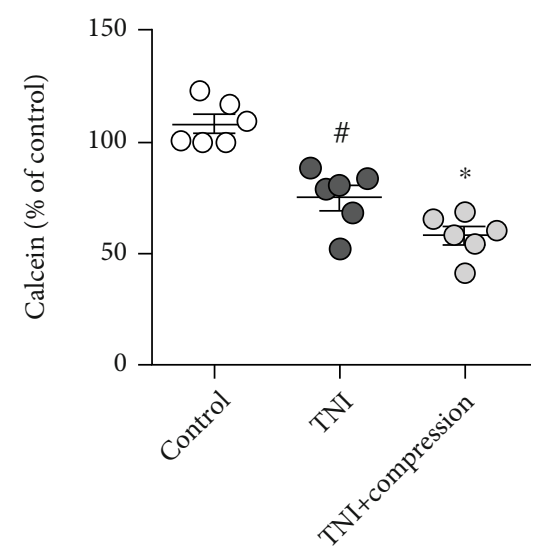

(c)

Figure 4: Compression aggravates neuronal injury after TNI in cortical neurons. (a) Typical pictures of H\&E staining show that compression aggravates neuronal loss after TNI. The dotted line indicates the edge of the scratch injury. The scale bar is $50 \mu \mathrm{m}$. (b) Compression increases LDH release after TNI. (c) Compression decreases calcein signal after TNI. The data was represented as means \pm SEM. ${ }^{\#} p<0.05$ vs. control group and ${ }^{*} p<0.05$ vs. TNI group.

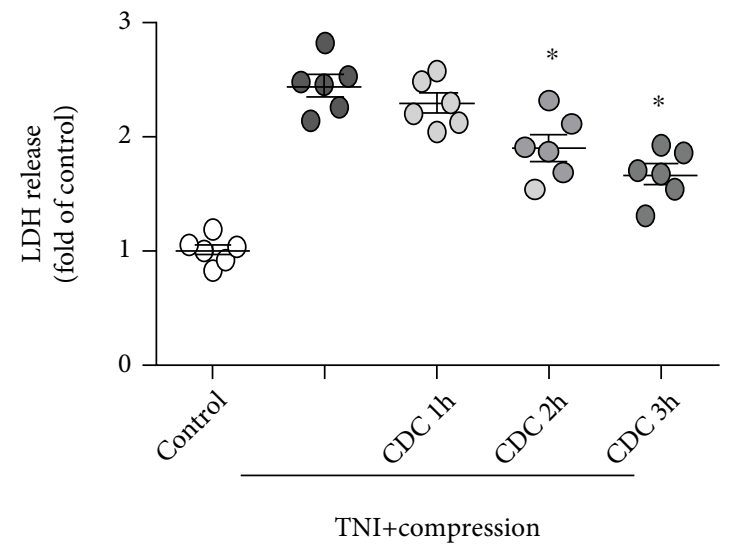

(a)

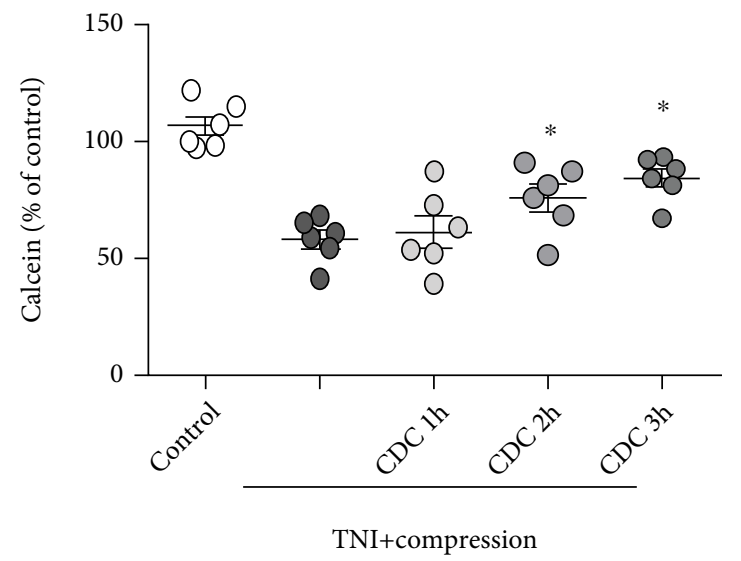

(b)

FIGURE 5: CDC attenuates compressive injury following TNI. (a) CDC for $2 \mathrm{~h}$ or $3 \mathrm{~h}$, but not $1 \mathrm{~h}$, attenuates compression-induced increase in $\mathrm{LDH}$ release following TNI. (b) CDC for $2 \mathrm{~h}$ or $3 \mathrm{~h}$, but not $1 \mathrm{~h}$, attenuates compression-induced decrease in calcein signal following TNI. The data was represented as means \pm SEM. ${ }^{*} p<0.05$ vs. TNI+compression group.

The results showed that TNI markedly decreased the calcein signal in cortical neurons, which was further aggravated by compression.

3.2. CDC Attenuates Compressive Injury following TNI. To mimic controlled decompression in vitro, the $0.5 \mathrm{MPa}$ pressure was gradually released within $1 \mathrm{~h}$ (CDC $1 \mathrm{~h}$ group), $2 \mathrm{~h}$ (CDC $2 \mathrm{~h}$ group), or $3 \mathrm{~h}$ (CDC $3 \mathrm{~h}$ group), while the pressure was completely released at once as control. The results showed that CDC $2 \mathrm{~h}$ and CDC $3 \mathrm{~h}$ significantly decreased the LDH release induced by TNI and compression, whereas
CDC $1 \mathrm{~h}$ had no effect (Figure 5(a)). In congruent, CDC $2 \mathrm{~h}$ and CDC $3 \mathrm{~h}$, but not CDC $1 \mathrm{~h}$, obviously increased the calcein signal after TNI and compression (Figure 5(b)). CDC $2 \mathrm{~h}$ was used in following experiments (represented as CDC).

3.3. CDC Alleviates Neuronal Necroptosis. Double staining with PI and DAPI was performed to detect necrotic cell death in cortical neurons (Figure 6(a)), and the results showed that the number of PI-positive cells was increased by TNI and compression but significantly decreased by CDC (Figure 6(b)). To investigate the role of necroptosis 


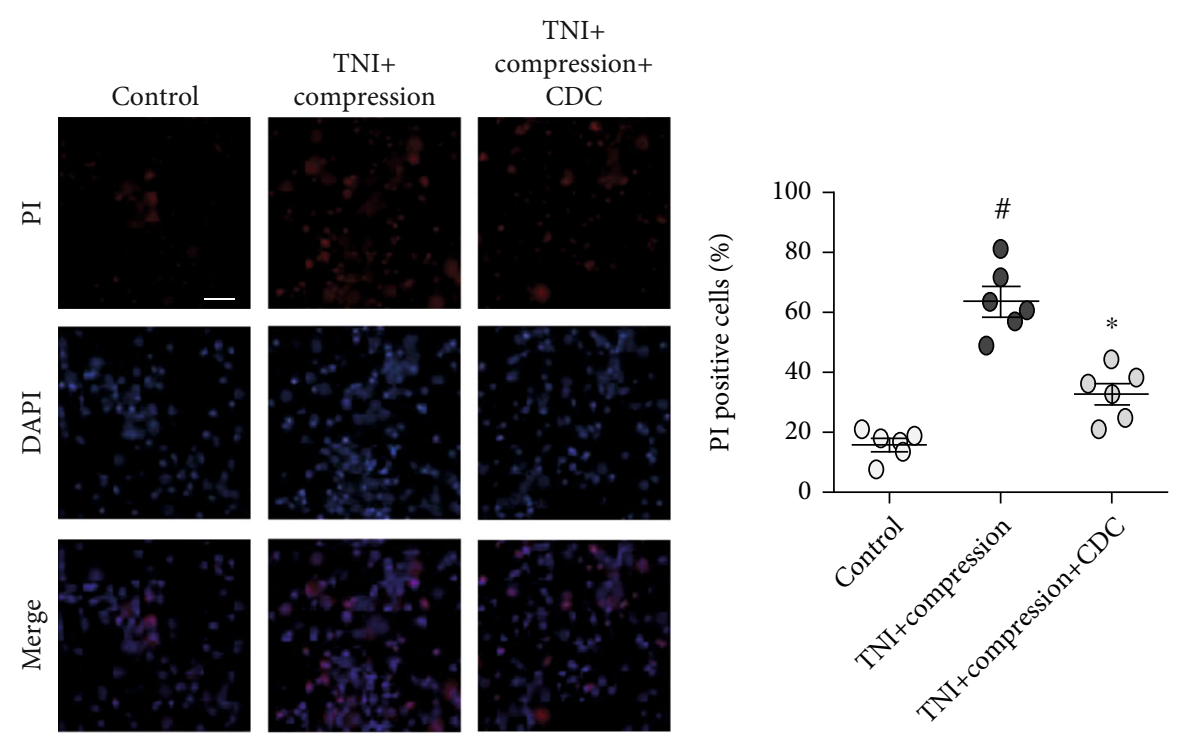

(a)

(b)

TNI+

compression+

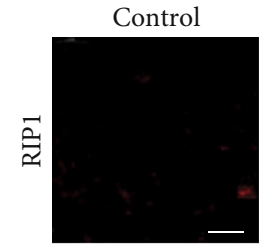

TNI+ compression

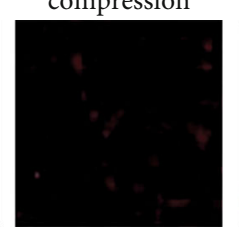

$\mathrm{CDC}$
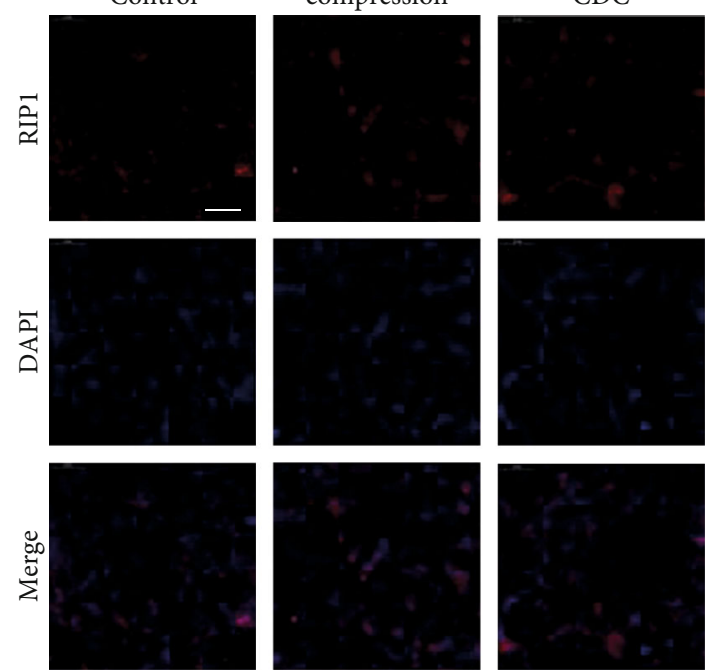

(c)

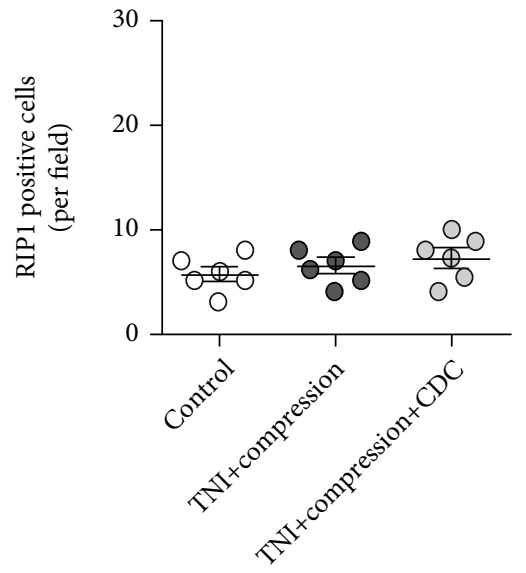

(d)

FIgure 6: Continued. 


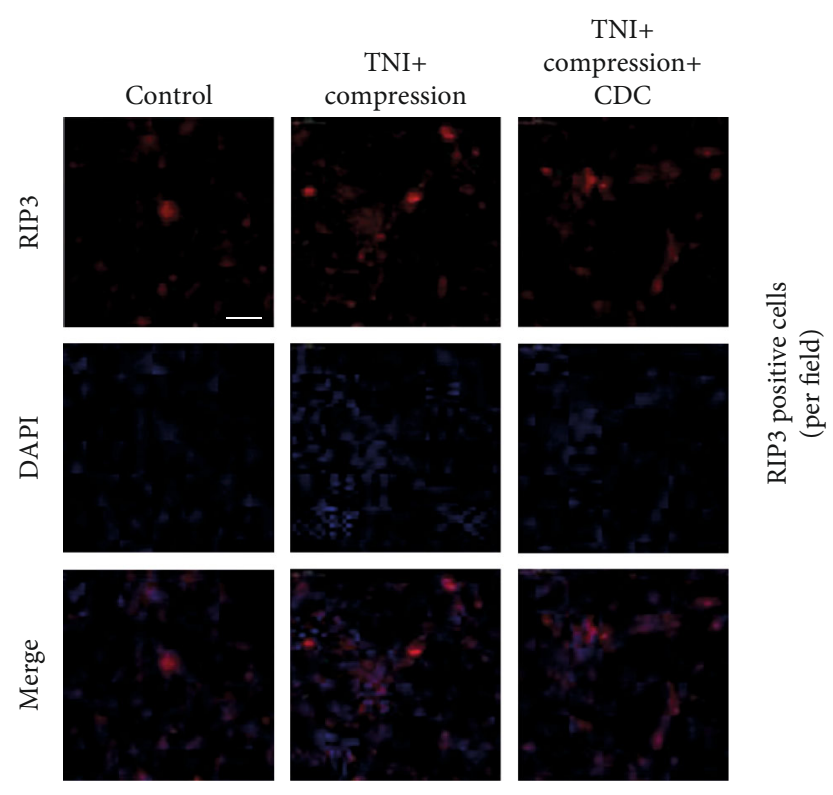

(e)

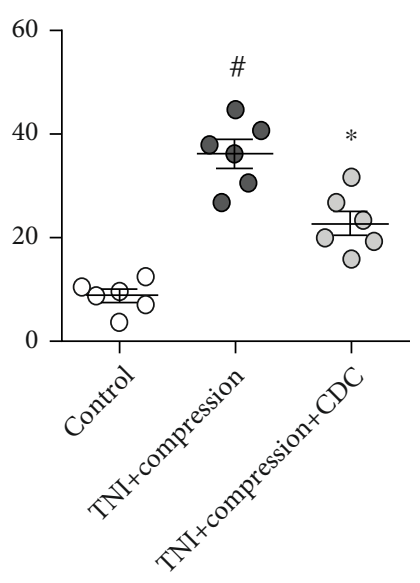

(f)

Figure 6: CDC alleviates neuronal necroptosis. (a, b) Typical pictures of PI staining (a) and quantification (b) show that CDC inhibits neuronal necrosis following TNI and compression. The scale bar is $50 \mu \mathrm{m}$. (c, d) Typical pictures of RIP1 staining (c) and quantification (d) show that CDC or compressive injury after TNI has no effect on RIP1 expression. The scale bar is $50 \mu \mathrm{m}$. (e, f) Typical pictures of RIP3 staining (e) and quantification ( $\mathrm{f}$ ) show that CDC attenuates RIP3 activation after TNI and compression. The scale bar is $50 \mu \mathrm{m}$. The data was represented as means \pm SEM. ${ }^{\#} p<0.05$ vs. control group and ${ }^{*} p<0.05$ vs. TNI+compression group.

in our in vitro experiments, we detected the expression of RIP1 (Figure 6(c)) and RIP3 (Figure 6(e)) using immunostaining. The results showed that neither TNI+compression nor CDC had effects on the number of RIP1-positive cells (Figure 6(d)). However, the increased number of RIP3positive cells was reduced by CDC (Figure 6(f)).

3.4. CDC Decreases the Levels of Inflammatory Cytokines. Necroptotic cell death was associated with inflammatory responses. Thus, we measured the levels of inflammatory cytokines in cortical neurons treated with TNI, compression, and/or CDC. The results showed that TNI increased the levels of TNF- $\alpha$ (Figure 7(a)) and IL- $1 \beta$ (Figure 7(b)), which were further increased by compression. However, these increases were significantly attenuated by CDC. The level of IL- 6 was increased by TNI and compression, which was decreased by CDC (Figure 7(c)). In contrast, the increased level of INF- $\gamma$ induced by TNI and compression was not altered by CDC (Figure 7(d)). As shown in Figure 7(e), the increased level of IL-18 after TNI and compression was markedly attenuated by CDC.

3.5. CDC Preserves Intracellular $\mathrm{Ca}^{2+}$ Homeostasis. Next, we performed $\mathrm{Ca}^{2+}$ imaging to examine the effect of TNI, compression, and CDC on calcium homeostasis (Figure 8(a)). The representative pictures of $\mathrm{Ca}^{2+}$ imaging are shown in Figure $8(\mathrm{~b})$. The results showed that TNI and compressioninduced $\mathrm{Ca}^{2+}$ overload were reduced by CDC (Figure 8(c)). The time of $\mathrm{Ca}^{2+}$ signaling to baseline in the CDC group was shorter than that in the TNI and compression group (Figure 8(d)).
3.6. CDC Activates TREK-1 Channels. To detect the potential involvement of TREK-1 in our findings, we performed immunostaining using the TREK-1 antibody (Figure 9(a)). The results showed that TNI did not change the fluorescence intensity of TREK-1 in cortical neurons, whereas compression significantly increased the TREK-1 signal after TNI (Figure 9(b)). The compression-induced increase in TREK1 fluorescence intensity after TNI was further enhanced by CDC. In addition, we investigated whether TNI or compression has effects on TREK-1 channel activity, which was detected by inside-out membrane patches at a physiological steady membrane voltage of $-40 \mathrm{mV}$ (Figure 9(c)). The results showed that the TREK-1 channel activation was markedly increased by compression, but not by TNI (Figure 9(d)).

\subsection{CDC Inhibits $\mathrm{Ca}^{2+}$ Responses via TREK-1 in Cortical} Neurons. To investigate the role of the TREK-1 channel in CDC-induced regulation of intracellular $\mathrm{Ca}^{2+}$ homeostasis after TNI, we repeated the $\mathrm{Ca}^{2+}$ imaging experiments using the TREK-1 blockers, spadin and SID1900. The results showed that the decreased intracellular $\mathrm{Ca}^{2+}$ concentration induced by compression after TNI was significantly preserved by both spadin $(1 \mu \mathrm{M}$, Figure 10(a)) and SID1900 $(30 \mu \mathrm{M}$, Figure $10(\mathrm{~b}))$.

3.8. Involvement of TREK-1 in CDC-Induced Protection In Vitro. Treatment with spadin and SID1900 (at the beginning of CDC) was used to investigate the involvement of TREK-1 in $\mathrm{CDC}$-induced protection and related mechanisms. The results showed that the $\mathrm{CDC}$-induced decrease in cytotoxicity, as evidenced by decreased $\mathrm{LDH}$ release, was prevented 


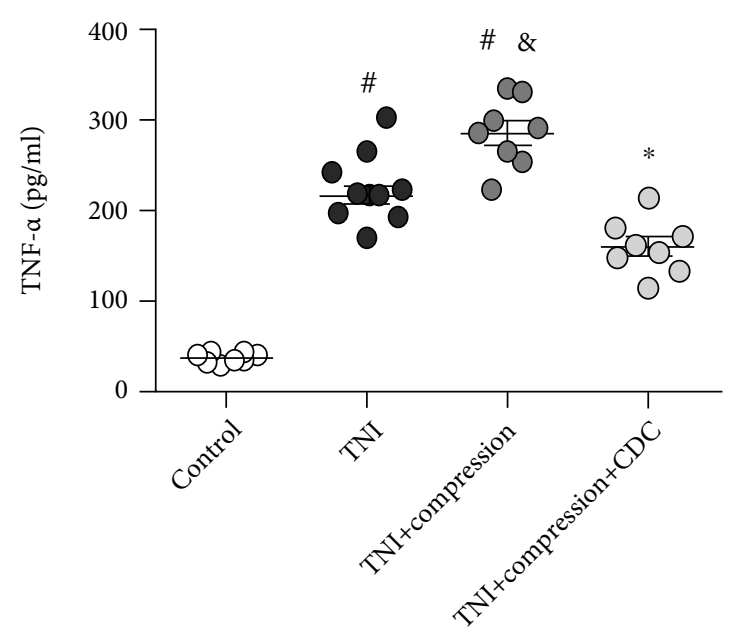

(a)

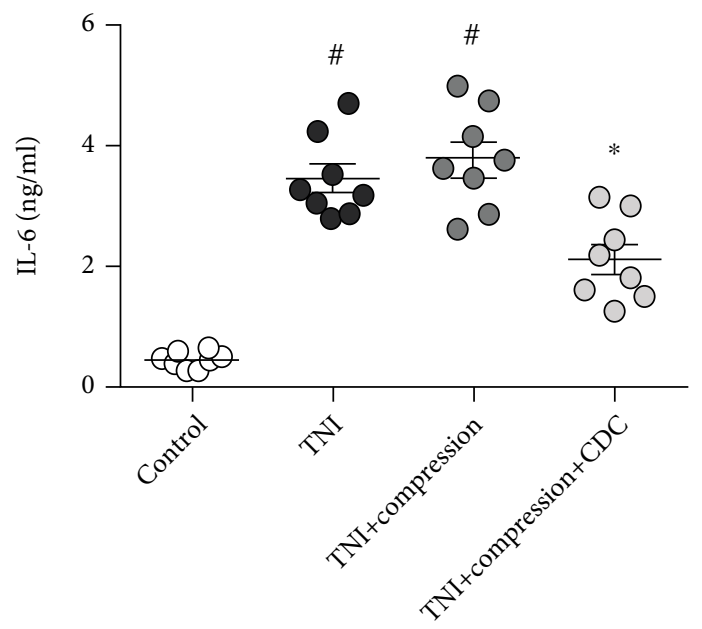

(c)

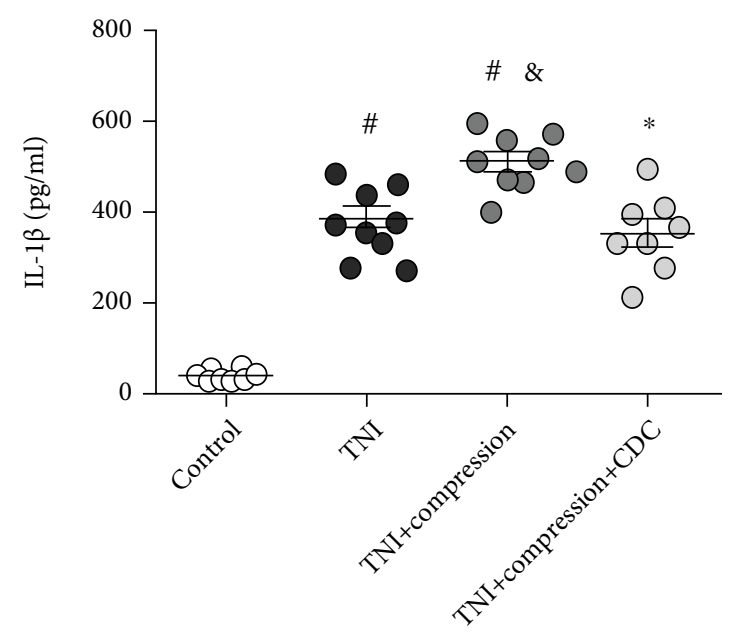

(b)

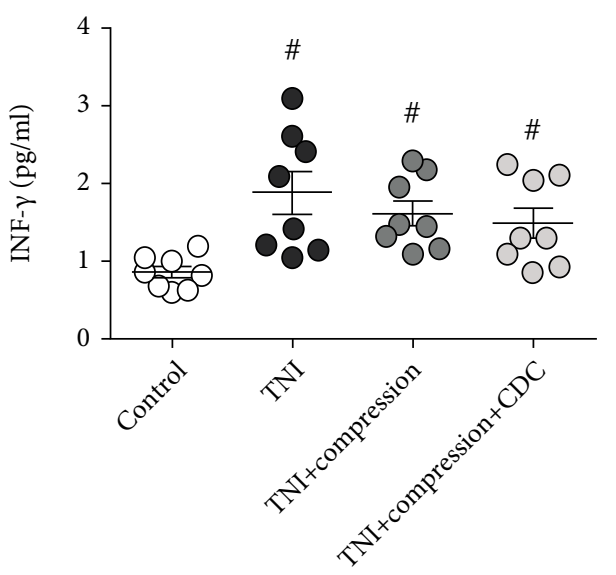

(d)

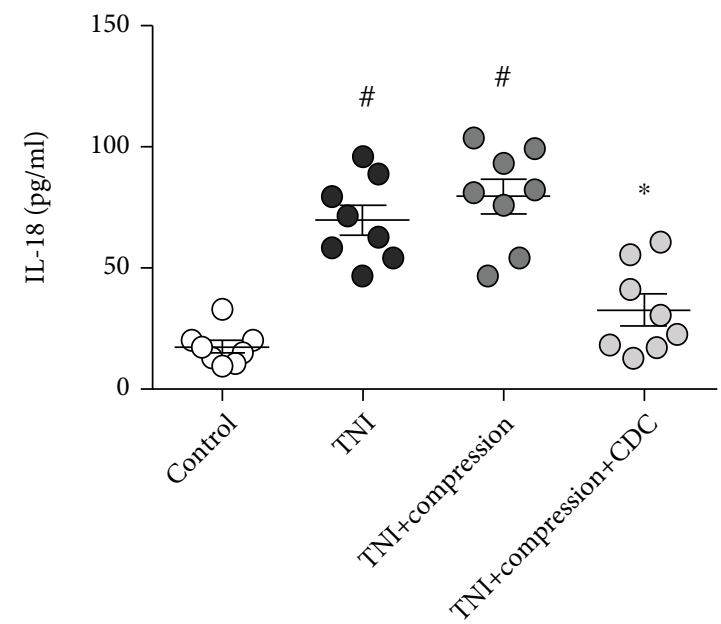

(e)

FIGURE 7: CDC decreases the levels of inflammatory cytokines. (a-c) ELISA assay shows that CDC decreases the levels of TNF- $\alpha$ (a), IL-1 $\beta$ (b), and IL-6 (c) after TNI and compression. (d) CDC has no effect on INF- $\gamma$ level in cortical neurons after TNI and compression treatment. (e) The TNI and compression-induced increase in IL-18 levels is attenuated by CDC. The data was represented as means \pm SEM. ${ }^{\#} p<0.05$ vs. control group, ${ }^{\&} p<0.05$ vs. TNI group, and ${ }^{*} p<0.05$ vs. TNI+compression group. 


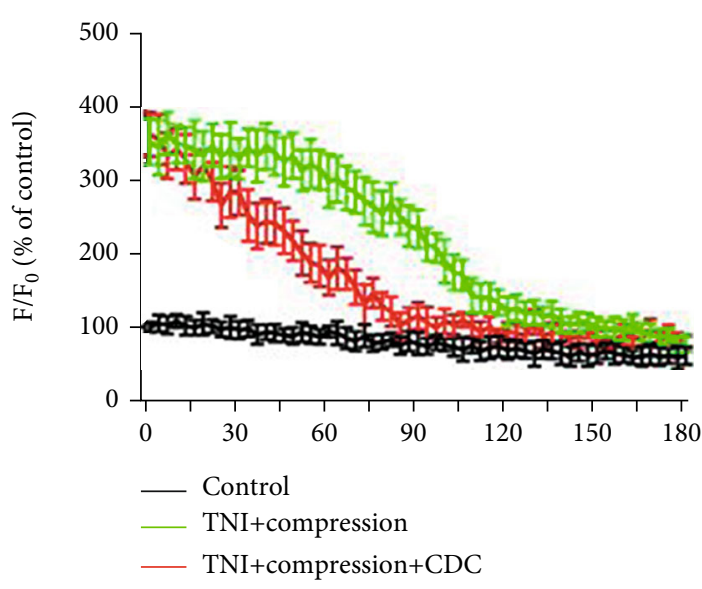

(a)
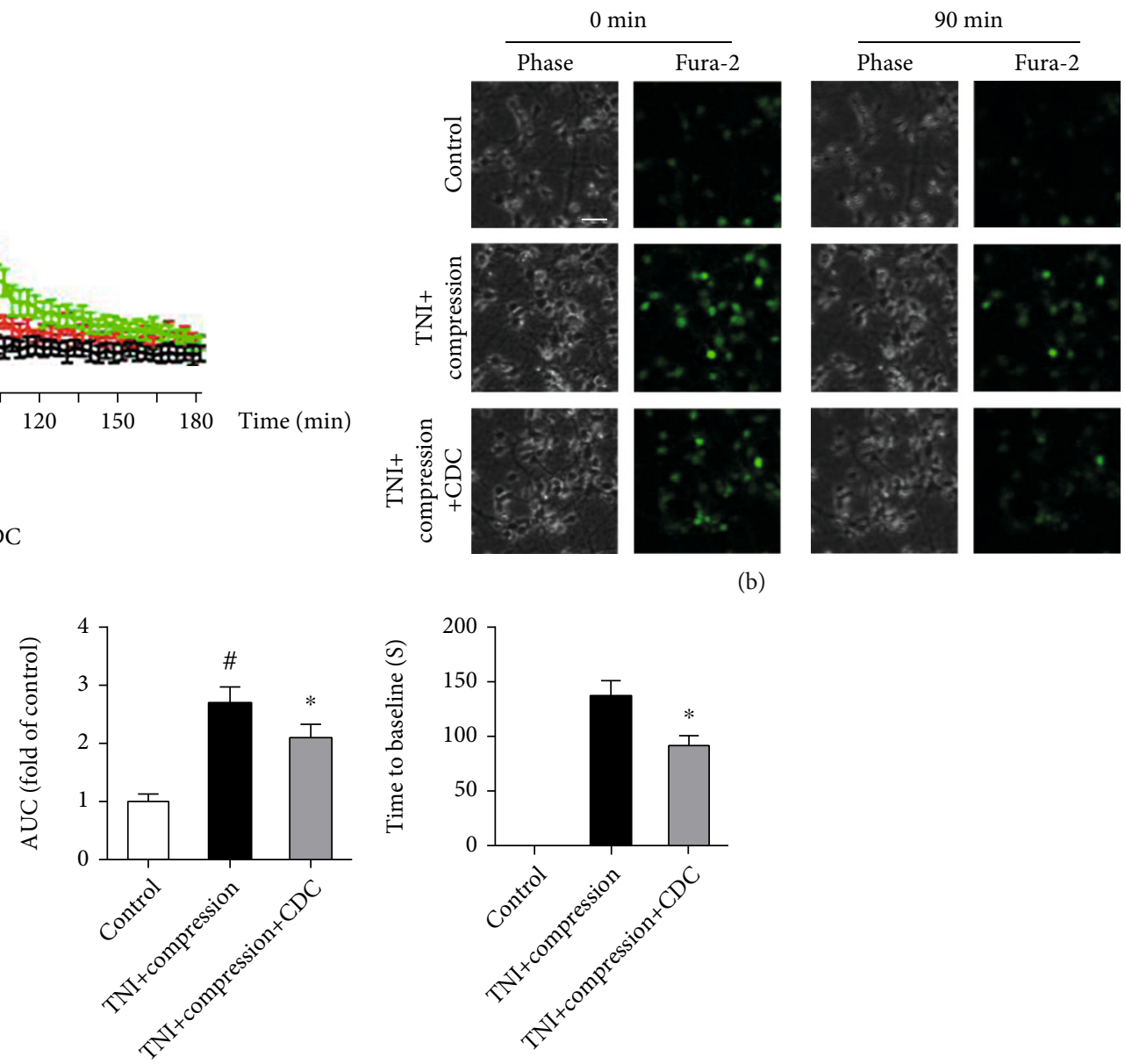

(c)

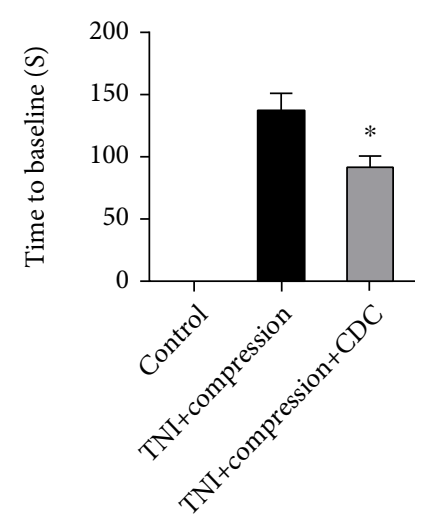

(d)

FIGURE 8: CDC preserves intracellular $\mathrm{Ca}^{2+}$ homeostasis. (a) $\mathrm{Ca}^{2+}$ imaging shows the intracellular $\mathrm{Ca}^{2+}$ levels up to 180 min after TNI and compression. (b) Typical pictures of $\mathrm{Ca}^{2+}$ imaging at 0 and $90 \mathrm{~min}$ following TNI and compression. The scale bar is $20 \mu \mathrm{m}$. (c) CDC attenuates intracellular $\mathrm{Ca}^{2+}$ overload up to 180 min following TNI and compression. (d) CDC reduces the time of $\mathrm{Ca}^{2+}$ levels to the baseline. The data was represented as means \pm SEM. ${ }^{*} p<0.05$ vs. control group and ${ }^{*} p<0.05$ vs. TNI+compression group.

by spadin and SID1900 (Figure 11(a)). Congruently, the CDC-induced increase in calcein signal (Figure 11(b)), as well as the CDC-induced decrease in the number of PIpositive cells (Figure 11(c)), was apparently reversed by spadin and SID1900. In addition, the CDC-induced inhibition of RIP3 expression was markedly attenuated by spadin, but not by SID1900 (Figure 11(d)).

3.9. CDC Attenuates Brain Damage after Traumatic Intracranial Hypertension. To confirm the above findings in in vivo conditions, we established a traumatic intracranial hypertension model in rats, and the horizontal and coronal schematic diagrams are shown in Figure 1(a). The results of the brain water content assay showed that CDC for $20 \mathrm{~min}$ or $30 \mathrm{~min}$, but not CDC for $10 \mathrm{~min}$, significantly reduced brain edema compared to the RDC group (Figure 1(b)). The neurological assay showed that CDC for $20 \mathrm{~min}$ or $30 \mathrm{~min}$, but not CDC for $10 \mathrm{~min}$, preserved locomotor function compared to RDC (Figure 1(c)). Next, we performed immunostaining using the NeuN antibody to detect the neuronal loss following traumatic intracranial hypertension (Figure 1(d)), and the results showed that CDC for $20 \mathrm{~min}$ or $30 \mathrm{~min}$, but not CDC for $10 \mathrm{~min}$, inhibited neuronal loss compared to RDC (Figure 1(e)). The $\mathrm{CDC}$ for $30 \mathrm{~min}$ was used in the following experiments.

3.10. CDC Alleviates Neuronal Necroptosis and Neuroinflammation In Vivo. To investigate the effects of CDC on neuronal necroptosis following traumatic intracranial hypertension, the expression of RIP1 (Figure 2(a)) and RIP3 (Figure 2(c)) on brain sections was detected by immunostaining using corresponding antibodies. The results showed that RDC and CDC both increased the expression of RIP1 compared to the sham group, but there is no difference between these two groups (Figure 2(b)). However, CDC markedly decreased the expression of RIP3 compared to RDC (Figure 2(d)). The activation of microglia was determined by immunostaining using the CD68 antibody (Figure 2(e)), and the results showed that microglial activation in the CDC group was lower than that in the RDC group (Figure 2(f)). 


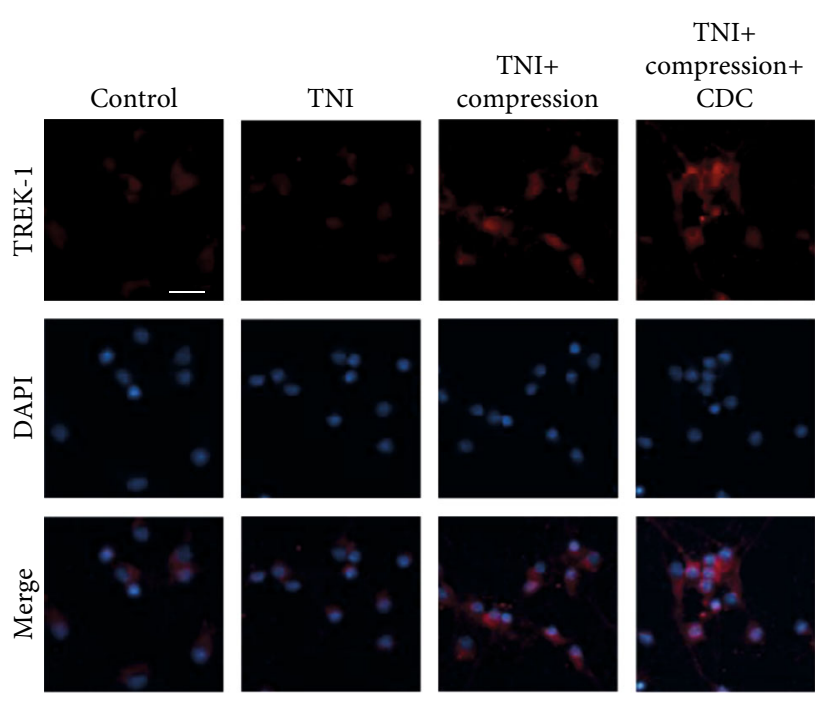

(a)
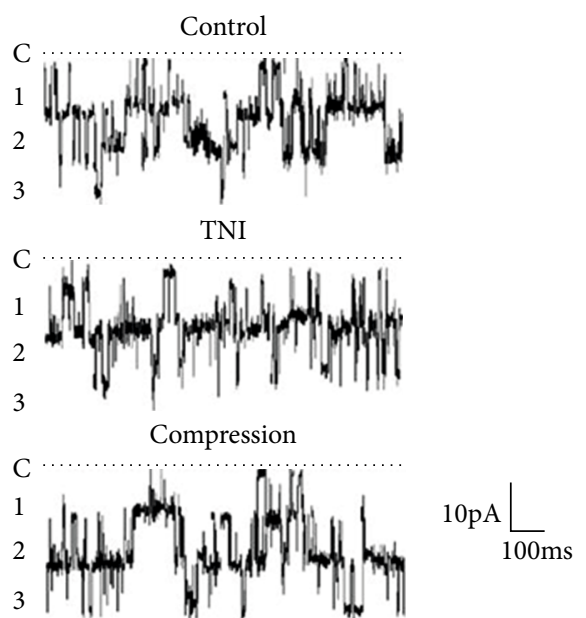

(c)

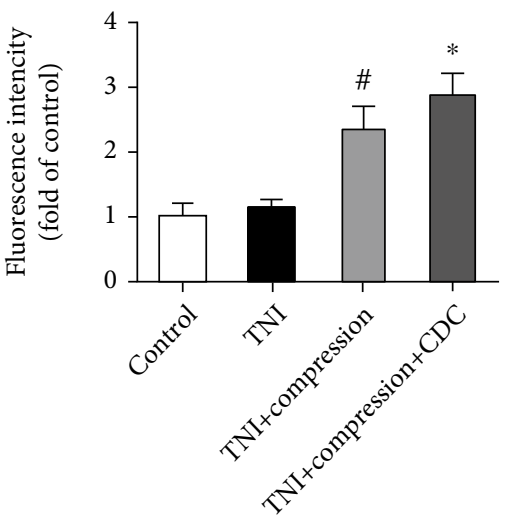

(b)

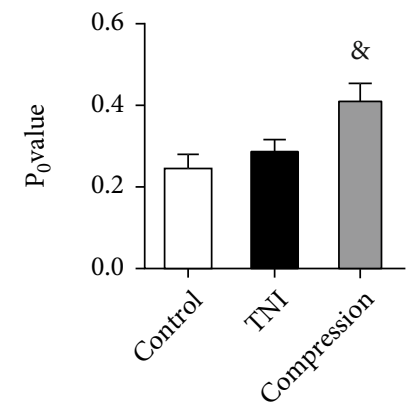

(d)

FIgURE 9: CDC activates TREK-1 channels. ( $a, b)$ Typical pictures of TREK-1 staining (a) and quantification (b) show that CDC further enhances the activation of TREK-1 channels following TNI and compression. The scale bar is $20 \mu \mathrm{m}$. (c, d) Typical recordings from the inside-out patch illustrating TREK-1 channel activation (c) and quantification (d) show that compression, but not TNI, activates TREK-1 channels in cortical neurons. The data was represented as means \pm SEM. ${ }^{*} p<0.05$ vs. TNI group, ${ }^{*} p<0.05$ vs. TNI+compression group, and ${ }^{\&} p<0.05$ vs. control group.

3.11. CDC Exerts Neuroprotective Effects via Activating TREK-1 In Vivo. We also performed immunostaining using the TREK-1 antibody in brain sections after traumatic intracranial hypertension (Figure 3(a)), and the results showed that RDC and CDC both increased the expression of TREK-1 with the higher levels of TREK-1 in the CDC group (Figure 4(b)). It was shown that the increased expression of TREK-1 was mainly in neurons, as evidenced by colocalization with NeuN staining (Figure 3(a)). To further confirm the involvement of TREK-1 in vivo, we repeated the brain water content assay (Figure 3(c)) and neurological function assay (Figure 3(d)) using spadin and SID9100. The result showed that the effects of CDC on brain edema and locomotor impairment were weakened by spadin and SID 9100 .

\section{Discussion}

Uncontrollable intracranial hypertension is one of the most important causes of death in TBI patients, but rapid decompression during the standard decompressive craniectomy surgery has been reported to be associated with many postoperative complications $[20,21]$. In this study, we demonstrated that compression aggravates neuronal injury after TNI in cortical neurons and identified CDC as an effective strategy to prevent this kind of neuronal injury. We found that (a) CDC ( $2 \mathrm{~h}$ and $3 \mathrm{~h}$ ) effectively reduces compressive neuronal damage following TNI, (b) CDC significantly inhibits neuronal necrosis and RIP3 activation;,(c) CDC markedly decreases the levels of inflammatory cytokines, (d) compression-induced intracellular $\mathrm{Ca}^{2+}$ overload is 


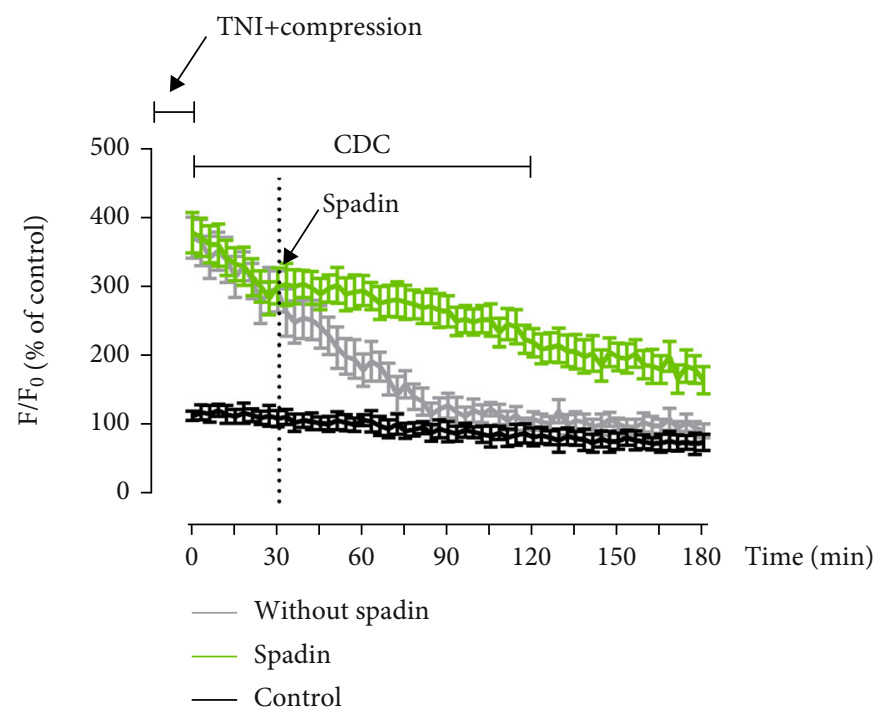

(a)

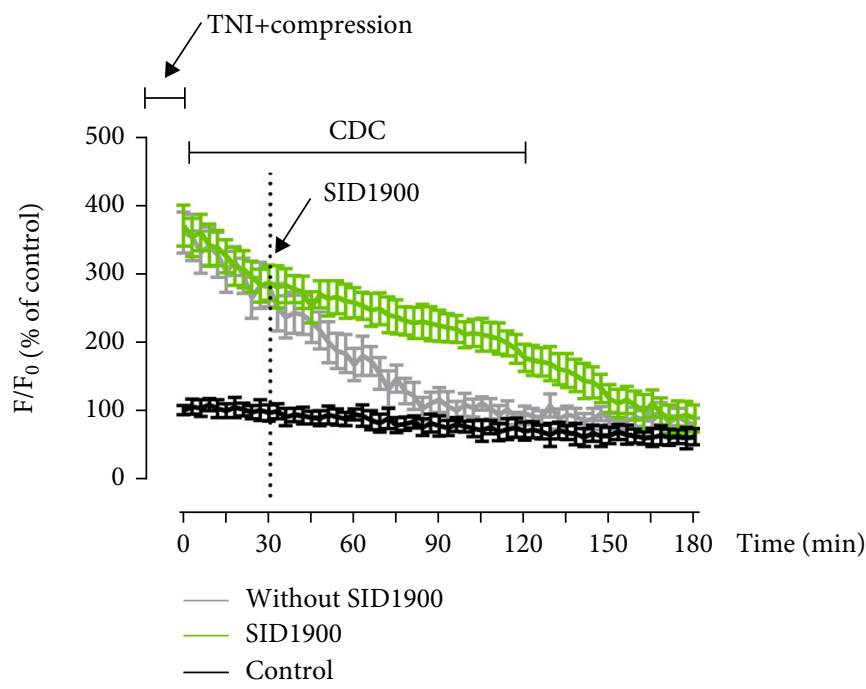

(b)

FIgURE 10: CDC inhibits $\mathrm{Ca}^{2+}$ responses via TREK-1. (a) $\mathrm{Ca}^{2+}$ imaging shows that $\mathrm{CDC}$-induced attenuation of intracellular $\mathrm{Ca}^{2+}$ overload was ablated by the TREK-1 blocker spadin. (b) $\mathrm{Ca}^{2+}$ imaging shows that $\mathrm{CDC}$-induced attenuation of intracellular $\mathrm{Ca}^{2+}$ overload was ablated by the TREK-1 blocker SID1900. The data was represented as means \pm SEM.

attenuated by CDC in vitro, (e) CDC enhances the expression and activity of TREK-1 channel induced by compression, (f) the CDC-induced effects on $\mathrm{Ca}^{2+}$ metabolism and neuronal injury following TNI and compression are decreased by TREK-1 blockers, (g) CDC (20 min and $30 \mathrm{~min}$ ) is effective in alleviating brain edema and locomotor impairment in vivo, (h) CDC inhibits neuronal necroptosis and microglial activation and increases TREK-1 expression, and (i) the CDC-induced protection in vivo is reversed by TREK-1 blockers.

At the beginning of the 19th century, decompressive craniectomy was firstly described by Kocher $\mathrm{T}$ and Harvey Cushing to treat patients with low Glasgow coma scale (GCS) score and dilated pupils following TBI. However, the standard procedure of rapid decompression was found to be associated with many intraoperative and postoperative complications, including delayed intracranial hematoma and diffuse brain swelling. In the past few decades, neurosurgeons have made a lot of improvements to the surgical procedures. Alves et al. used "Basal durotomy", a novel design of dural opening with a "reversed U-shaped" durotomy incision, to minimize the risk of massive intraoperative swelling [22]. In 2013, decompressive craniectomy with multi-dural stabs (also called SKIMS technique) was introduced to be effective in increasing survival of low GCS score and severe TBI patients with acute subdural hematoma [23]. Our previous studies showed that decompressive craniectomy using the CDC strategy, a method to gradually reduce the intracranial pressure (ICP) with the ventricular or brain tissue ICP detector, significantly improved outcomes and reduced complications in severe TBI patients [5]. Here, we used the in vitro model to confirmed the effectiveness of CDC in 


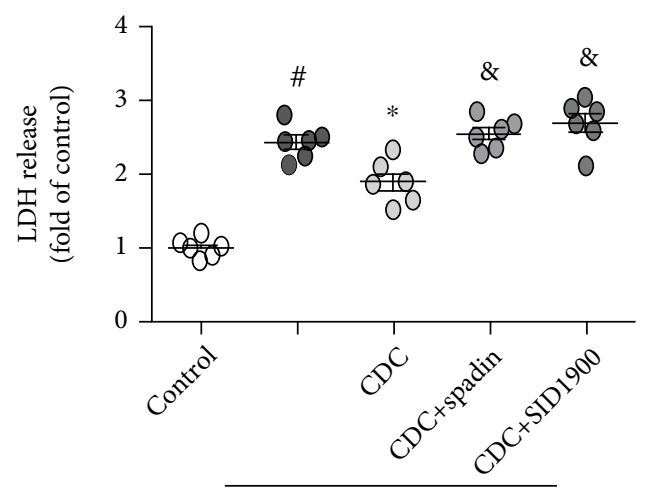

TNI+compression

(a)

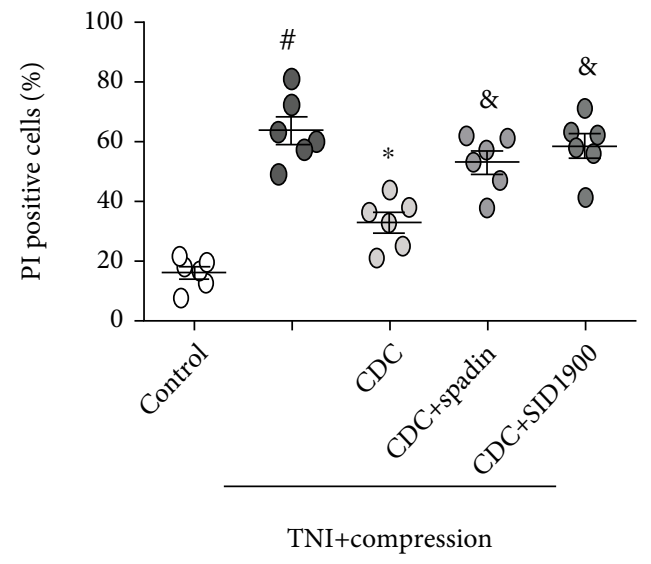

(c)

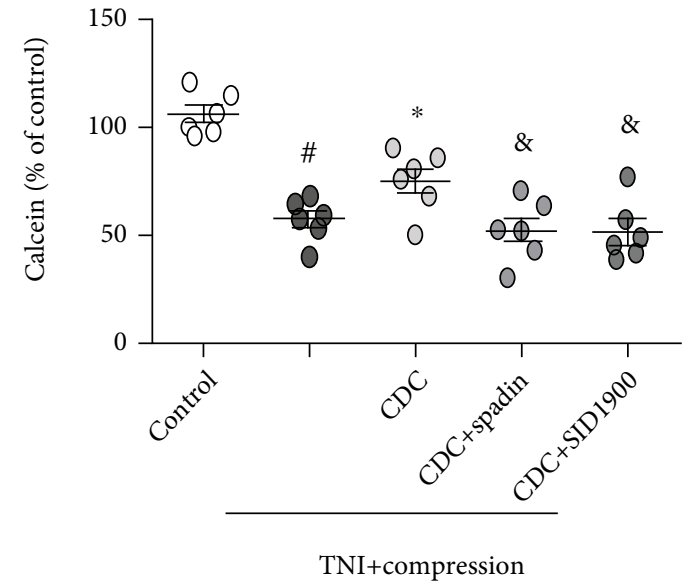

(b)

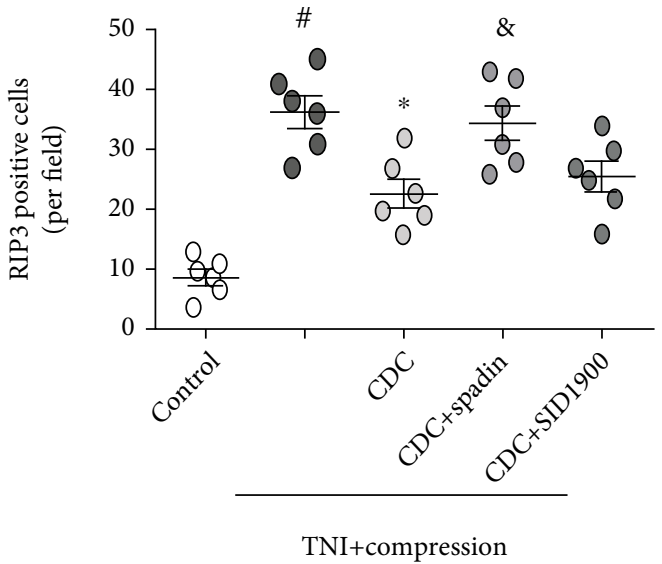

(d)

FIGURE 11: Involvement of TREK-1 in CDC-induced protection. (a) The CDC-induced decrease in LDH release was prevented by spadin and SID1900. (b) The CDC-induced increase in calcein signal was prevented by spadin and SID1900. (c) The CDC-induced inhibition of RIP3 activation was prevented by spadin and SID1900. (d) The CDC-induced inhibition of neuronal necrosis was prevented by spadin, but not by SID1900. The data was represented as means \pm SEM. ${ }^{\#} p<0.05$ vs. control group, ${ }^{*} p<0.05$ vs. TNI+compression group, and $\&_{p}<0.05$ vs. $\mathrm{CDC}+\mathrm{TNI}+$ compression group.

TNI- and compression-treated cortical neurons. Our results showed that CDC for 2 and $3 \mathrm{~h}$ markedly reduced LDH release and increased calcein signal, which were accompanied by inhibited neuronal death. In addition, these protective effects were also confirmed in an in vivo traumatic intracranial hypertension model in rats with the CDC procedure for $20 \mathrm{~min}$ or $30 \mathrm{~min}$. Thus, CDC could be an effective strategy for the treatment of compressive neuronal injury following TBI both in vitro and in vivo.

Regulated neuronal death is thought to be an ideal therapeutic target for neurological disorders. However, the primary brain damage following TBI is mainly due to neuronal necrosis, an uncontrollable cell death characterized by ATP depletion, intracellular organelle swelling, and loss of membrane integrity [24-26]. Necroptosis is the first form of programmed necrosis with an important role in both physiological and pathological conditions [27]. More recently, the necroptosis inhibitor necrostatin-1 was predicted to have the potential to protect against the complications of coronavirus disease 2019 (COVID-19) [28]. In addition, necroptosis has been demonstrated to contribute to the delayed neuronal death in multiple neurological disorders, ranging from chronic neurodegenerative disorders such as Alzheimer's disease (AD) and Parkinson's disease (PD) to acute insults, including stroke and TBI $[29,30]$. The necroptosis inhibitors were found to exert neuroprotective effects against TBI [31], and blocking necroptotic neuronal death was shown to mediate the therapeutic potential of many treatments for TBI, such as hypothermia [32]. In this study, increased number of PI-positive cells was found in TNI- and compression-treated neurons, confirming the involvement of necroptosis in our in vitro traumatic intracranial hypertension conditions. The necroptotic pathway depends on the assembly of the apical protein kinases, receptor-interacting protein kinase 1 (RIP1) and RIP3, to form the high molecular weight complex necrosome, which in turn governs the oligomerization and translocation of the cell death executor mixed lineage kinase domain-like (MLKL) [33]. Intriguingly, our results showed that TNI and compression increased RIP3 (but not RIP1) activation, 
which was partially prevented by CDC. In addition, increased expression of RIP1 and RIP3 was found after traumatic intracranial hypertension in vivo, while CDC only decreased RIP3 expression. These data indicated that CDC-induced inhibition of neuronal necroptosis might be mediated by a RIP1-independent mechanism, which was also previously reported $[34,35]$.

As the stretch-dependent $\mathrm{K}^{+}$channels (with TREK-2 and TRAAK), TREK-1 was originally described in rat cardiac ventricular muscle, where mechanical stretch could increase its expression in mechanoelectrical coupling [36]. Following researches demonstrated that the mechanogating activity of TREK-1 channels was also important for the sensation of pain in sensory neurons [37]. Thus, to determine the potential involvement of TREK-1, we detected the expression and activity of TREK-1. We found that TNI had no effect on the expression and activity of TREK-1, but compression significantly potentiated TREK-1 activation, which was prolonged by CDC strategy. Immunostaining results in brain sections also showed that CDC markedly increased the expression of TREK-1 in neurons. The TREK-1-induced $\mathrm{K}^{+}$efflux could counter-balance the detrimental arrhythmogenic cation influx through nonselective cation channels, such as $\mathrm{Na}^{+}$channels [38]. In addition, TREK-1 was demonstrated to mediate the fast glutamate release in astrocytes via the activation of G-proteincoupled receptors (GPCRs) [39]. These data suggest that TREK-1 might be involved in glutamate-associated neurotoxicity. To confirm this hypothesis, we used two TREK-1 specific inhibitors, spadin and SID1900. Spadin is a synthetic peptide derived from sortilin which was shown to inhibit TREK-1 with high affinity [11]. The small molecule SID1900 was found to inhibit TREK-1 with an IC $_{50}$ of $29.72 \mu \mathrm{M}$, comparing that of spadin is $40 \mathrm{nM}$ [40]. The results showed that $\mathrm{CDC}$-induced effects on $\mathrm{Ca}^{2+}$ regulation, neuronal injury, as well as the in vivo protective effects, were all partially reversed by spadin and SID1900, confirming the role of TREK-1 in CDC-induced neuroprotection. Intriguingly, a previous study has reported the negative results of TBI-related brain damage in mice lacking TREK-1 [41]. However, deficiency of TREK-1 was shown to exacerbate BBB injury and neuroinflammation in intracranial hemorrhage [42]. The role of TREK-1 in neuronal injury seems to be controversial in different experimental models, where mechanical force could be an important factor.

As the most abundant divalent cation in mammalian cells, $\mathrm{Ca}^{2+}$ serves as the key intracellular signaling molecule with a striking 10000 -fold concentration gradient larger than other ions, including $\mathrm{Na}^{+}$and $\mathrm{K}^{+}$[43]. The physiological function of $\mathrm{Ca}^{2+}$ in the central nervous system depends on where, when, and how it enters and exits the neurons and glial cells. Increasing evidence indicate that dysfunction of intracellular $\mathrm{Ca}^{2+}$ homeostasis is involved in TBI-induced brain damage, and many $\mathrm{Ca}^{2+}$ channel blockers are thought to exert neuroprotective potential [44]. Our previous study showed that compression resulted in intracellular $\mathrm{Ca}^{2+}$ overload via promoting intracellular $\mathrm{Ca}^{2+}$ release in primary cultured cortical neurons [16]. Here, we detected the changes in intracellular $\mathrm{Ca}^{2+}$ concentrations using $\mathrm{Ca}^{2+}$ imaging, and the results showed that compressive injury following TNI caused an increase in the $F / F_{0}$ signal, which was attenuated by CDC. A previous study showed that TREK-1 gene knockout impairs neuronal excitability, synaptic plasticity, and cognitive function [12]. The activity of TREK-1 channels can be regulated by various chemical stimuli, including Gq-coupled group I mGluRs, mGluR1, and mGluR5, which enhance the release of $\mathrm{Ca}^{2+}$ from the intracellular calcium stores by stimulating 1,4,5-trisphosphate receptors $\left(\mathrm{IP}_{3} \mathrm{R}\right)$ on the endoplasmic reticulum (ER) membrane [45]. Thus, we repeated the $\mathrm{Ca}^{2+}$ imaging experiments using TREK-1 inhibitors, and the intracellular $\mathrm{Ca}^{2+}$ overload was found to be prolonged after blocking TREK-1 activation. Congruently, TREK-1 was shown to regulate pressure sensitivity and $\mathrm{Ca}^{2+}$ signaling in trabecular meshwork cells [46], and the TREK-1-specific blocker spadin was found to potentiate $\mathrm{Ca}^{2+}$ influx and insulin secretion in pancreatic beta cells [47]. These data strongly suggest that the TREK-1mediated neuroprotective mechanism was associated with the preservation of intracellular $\mathrm{Ca}^{2+}$ homeostasis, possibly via regulating $\mathrm{Ca}^{2+}$ release form intracellular $\mathrm{Ca}^{2+}$ stores, which needs to be further determined.

\section{Conclusions}

In summary, our present data indicate that $\mathrm{CDC}$ for $2 \mathrm{~h}$ or $3 \mathrm{~h}$ in vitro and CDC for $20 \mathrm{~min}$ or $30 \mathrm{~min}$ in vivo exert neuroprotective effects. The potential underlying mechanisms involve in TREK-1 mediated regulation of intracellular $\mathrm{Ca}^{2+}$ homeostasis and inhibition of neuronal necroptosis.

\section{Data Availability}

The research data used to support the findings of this study are included within the article.

\section{Ethical Approval}

All experimental procedures used in this study were approved by the Ethics Review Committee of Anhui Medical University (Hefei, China).

\section{Conflicts of Interest}

The authors declare that they have no conflict of interest.

\section{Authors' Contributions}

YHW and LKY conceived and designed the study. TC, XQ, and JZ performed the experiments. JZ analyzed the data. TC wrote the manuscript. YHW and LKY reviewed and revised the manuscript and supervised the study. All authors read and approved the manuscript. Tao Chen and Xiao Qian contributed equally to this work.

\section{Acknowledgments}

This work was supported by the National Natural Science Foundation of China (No. 81701932, No. 81871589, and No. 82072168), the Natural Science Foundation of Jiangsu 
Province (No. MS2022200), the Major Scientific Research Project of the Wuxi Health Commission (No. Z202001), the Top Talent Support Program for Young and MiddleAged People of the Wuxi Health Committee (BJ2020118), the Translational Medicine Research Major Project of the Wuxi Health Commission (No. ZH201901), the China Postdoctoral Science Foundation Funded Project (No. 2019M651803), the Key Scientific Research Project of the Jiangsu Health Commission (No. K2019018), the Research Project of the Wuxi Health Commission (No. MS201910), and the Logistics Scientific Research Project of the PLA (No. CLB20J027).

\section{References}

[1] Z. Rossini, F. Nicolosi, A. G. Kolias, P. J. Hutchinson, P. de Sanctis, and F. Servadei, "The history of decompressive craniectomy in traumatic brain injury," Frontiers in Neurology, vol. 10, p. 458, 2019.

[2] N. Fatima, G. al Rumaihi, A. Shuaib, and M. Saqqur, "The role of decompressive craniectomy in traumatic brain injury: a systematic review and meta-analysis," Asian J Neurosurg, vol. 14, no. 2, pp. 371-381, 2019.

[3] A. G. Kolias, H. Adams, I. Timofeev et al., "Decompressive craniectomy following traumatic brain injury: developing the evidence base," British Journal of Neurosurgery, vol. 30, no. 2, pp. 246-250, 2016.

[4] H. Guan, C. Zhang, T. Chen et al., "Controlled decompression attenuates brain injury in a novel rabbit model of acute intracranial hypertension," Medical Science Monitor, vol. 25, pp. 9776-9785, 2019.

[5] J. Chen, M. Li, L. Chen et al., "The effect of controlled decompression for severe traumatic brain injury: a randomized controlled trial," Frontiers in Neurology, vol. 11, p. 107, 2020.

[6] Y. Wang, W. Chunli, Y. Likun et al., "Controlled decompression for the treatment of severe head injury: a preliminary study," Turkish Neurosurgery, vol. 24, no. 2, pp. 214-220, 2014.

[7] R. Santos, O. Ursu, A. Gaulton et al., "A comprehensive map of molecular drug targets," Nature Reviews Drug Discovery, vol. 16, no. 1, pp. 19-34, 2017.

[8] C. Heurteaux, N. Guy, C. Laigle et al., "TREK-1, a K+ channel involved in neuroprotection and general anesthesia," The EMBO Journal, vol. 23, no. 13, pp. 2684-2695, 2004.

[9] M. Fink, F. Duprat, F. Lesage et al., "Cloning, functional expression and brain localization of a novel unconventional outward rectifier $\mathrm{K}+$ channel," The EMBO Journal, vol. 15, no. 24, pp. 6854-6862, 1996.

[10] E. Honore, "The neuronal background $\mathrm{K}_{2 \mathrm{P}}$ channels: focus on TREK1," Nature Reviews Neuroscience, vol. 8, no. 4, pp. 251$261,2007$.

[11] A. Djillani, J. Mazella, C. Heurteaux, and M. Borsotto, "Role of TREK-1 in Health and Disease focus on the central nervous system," Frontiers in Pharmacology, vol. 10, p. 379, 2019.

[12] W. Wang, C. M. Kiyoshi, Y. du et al., "TREK-1 null impairs neuronal excitability, synaptic plasticity, and cognitive function," Molecular Neurobiology, vol. 57, no. 3, pp. 1332-1346, 2020.

[13] X. Wu, Y. Liu, X. Chen et al., "Involvement of TREK-1 activity in astrocyte function and neuroprotection under simulated ischemia conditions," Journal of Molecular Neuroscience, vol. 49, no. 3, pp. 499-506, 2013.
[14] T. Chen, F. Fei, X. F. Jiang et al., "Down-regulation of Homer1b/c attenuates glutamate-mediated excitotoxicity through endoplasmic reticulum and mitochondria pathways in rat cortical neurons," Free Radical Biology and Medicine, vol. 52, no. 1, pp. 208-217, 2012.

[15] W. Rao, L. Zhang, C. Peng et al., "Downregulation of STIM2 improves neuronal survival after traumatic brain injury by alleviating calcium overload and mitochondrial dysfunction," Biochimica et Biophysica Acta (BBA) - Molecular Basis of Disease, vol. 1852, no. 11, pp. 2402-2413, 2015.

[16] T. Chen, J. Zhu, Y. H. Wang, and C. H. Hang, "ROS-mediated mitochondrial dysfunction and ER stress contribute to compression-induced neuronal injury," Neuroscience, vol. 416, pp. 268-280, 2019.

[17] T. Chen, J. Zhu, Y. H. Wang, and C. H. Hang, "Arc silence aggravates traumatic neuronal injury via mGluR1-mediated ER stress and necroptosis," Cell Death \& Disease, vol. 11, no. 1 , p. $4,2020$.

[18] T. Chen, W. B. Liu, X. D. Chao et al., "Salvianolic acid B attenuates brain damage and inflammation after traumatic brain injury in mice," Brain Research Bulletin, vol. 84, no. 2, pp. 163-168, 2011.

[19] R. Campos-Pires, H. Onggradito, E. Ujvari et al., "Xenon treatment after severe traumatic brain injury improves locomotor outcome, reduces acute neuronal loss and enhances early beneficial neuroinflammation: a randomized, blinded, controlled animal study," Critical Care, vol. 24, no. 1, p. 667, 2020.

[20] A. F. de Andrade, R. L. Amorim, D. J. F. Solla et al., "New technique for surgical decompression in traumatic brain injury: merging two concepts to prevent early and late complications of unilateral decompressive craniectomy with dural expansion," International Journal of Burns and Trauma, vol. 10, no. 3, pp. 76-80, 2020.

[21] P. Grille and N. Tommasino, "Decompressive craniectomy in severe traumatic brain injury: prognostic factors and complications," Revista Brasileira de Terapia Intensiva, vol. 27, no. 2, pp. 113-118, 2015.

[22] O. L. Alves and R. Bullock, ""Basal durotomy" to prevent massive intra-operative traumatic brain swelling," Acta Neurochirurgica, vol. 145, no. 7, pp. 583-586, 2003.

[23] A. R. Bhat, A. R. Kirmani, and M. A. Wani, "Decompressive craniectomy with multi-dural stabs - a combined (SKIMS) technique to evacuate acute subdural hematoma with underlying severe traumatic brain edema," Asian Journal of Neurosurgery, vol. 8, no. 1, pp. 15-20, 2013.

[24] M. Desai and A. Jain, "Neuroprotection in traumatic brain injury," J Neurosurg Sci, vol. 62, no. 5, pp. 563-573, 2018.

[25] D. J. Loane, B. A. Stoica, and A. I. Faden, "Neuroprotection for traumatic brain injury," Handbook of Clinical Neurology, vol. 127, pp. 343-366, 2015.

[26] R. Watzlawick, D. W. Howells, and J. M. Schwab, "Neuroprotection after traumatic brain injury," JAMA Neurology, vol. 73, no. 2, pp. 149-150, 2016.

[27] L. Della Torre, A. Nebbioso, H. G. Stunnenberg, J. H. A. Martens, V. Carafa, and L. Altucci, "The role of necroptosis: biological relevance and its involvement in cancer," Cancers (Basel), vol. 13, no. 4, p. 684, 2021.

[28] L. Cao and W. Mu, "Necrostatin-1 and necroptosis inhibition: pathophysiology and therapeutic implications," Pharmacological Research, vol. 163, p. 105297, 2021. 
[29] Z. Bao, L. Fan, L. Zhao et al., "Silencing of A20 aggravates neuronal death and inflammation after traumatic brain injury: a potential trigger of necroptosis," Frontiers in Molecular Neuroscience, vol. 12, p. 222, 2019.

[30] H. Ni, Q. Rui, X. Lin, D. Li, H. Liu, and G. Chen, “2-BFI provides neuroprotection against inflammation and necroptosis in a rat model of traumatic brain injury," Frontiers in Neuroscience, vol. 13, p. 674, 2019.

[31] Y. Q. Wang, L. Wang, M. Y. Zhang et al., "Necrostatin-1 suppresses autophagy and apoptosis in mice traumatic brain injury model," Neurochemical Research, vol. 37, no. 9, pp. 1849-1858, 2012.

[32] T. Liu, D. X. Zhao, H. Cui et al., "Therapeutic hypothermia attenuates tissue damage and cytokine expression after traumatic brain injury by inhibiting necroptosis in the rat," Scientific Reports, vol. 6, no. 1, 2016.

[33] Y. Meng, J. J. Sandow, P. E. Czabotar, and J. M. Murphy, "The regulation of necroptosis by post-translational modifications," Cell Death \& Differentiation, vol. 28, no. 3, pp. 861-883, 2021.

[34] J. P. Ingram, R. J. Thapa, A. Fisher et al., "ZBP1/DAI drives RIPK3-mediated cell death induced by IFNs in the absence of RIPK1," The Journal of Immunology, vol. 203, no. 5, pp. 1348-1355, 2019.

[35] D. M. Moujalled, W. D. Cook, T. Okamoto et al., "TNF can activate RIPK3 and cause programmed necrosis in the absence of RIPK1," Cell Death \& Disease, vol. 4, no. 1, article e465, 2013.

[36] F. Zhao, L. Dong, L. Cheng, Q. Zeng, and F. Su, "Effects of acute mechanical stretch on the expression of mechanosensitive potassium channel TREK-1 in rat left ventricle," Journal of Huazhong University of Science and Technology, vol. 27, no. 4, pp. 385-387, 2007.

[37] A. Mathie and E. L. Veale, "Two-pore domain potassium channels: potential therapeutic targets for the treatment of pain," Pflügers Archiv-European Journal of Physiology, vol. 467, no. 5, pp. 931-943, 2015.

[38] K. Monaghan, S. A. Baker, L. Dwyer et al., "The stretchdependent potassium channel TREK-1 and its function in murine myometrium," The Journal of physiology, vol. 589, no. 5, pp. 1221-1233, 2011.

[39] D. H. Woo, K. S. Han, J. W. Shim et al., “TREK-1 and Best1 channels mediate fast and slow glutamate release in astrocytes upon GPCR activation," Cell, vol. 151, no. 1, pp. 25-40, 2012.

[40] A. Djillani, M. Pietri, J. Mazella, C. Heurteaux, and M. Borsotto, "Fighting against depression with TREK-1 blockers: past and future. A focus on spadin," Pharmacology \& Therapeutics, vol. 194, pp. 185-198, 2019.

[41] K. Namiranian, C. D. Brink, J. C. Goodman, C. S. Robertson, and R. M. Bryan Jr., "Traumatic brain injury in mice lacking the K channel, TREK-1," Journal of Cerebral Blood Flow \& Metabolism, vol. 31, no. 3, pp. e1-e6, 2011.

[42] Y. Fang, Y. Tian, Q. Huang et al., "Deficiency of TREK-1 potassium channel exacerbates blood-brain barrier damage and neuroinflammation after intracerebral hemorrhage in mice," Journal of Neuroinflammation, vol. 16, no. 1, p. 96, 2019.

[43] A. De Loof, “The essence of female-male physiological dimorphism: differential $\mathrm{Ca}^{2+}$-homeostasis enabled by the interplay between farnesol-like endogenous sesquiterpenoids and sexsteroids? The Calcigender paradigm," General And Comparative Endocrinology, vol. 211, pp. 131-146, 2015.
[44] S. L. Zup and A. M. Madden, "Gonadal hormone modulation of intracellular calcium as a mechanism of neuroprotection," Frontiers in Neuroendocrinology, vol. 42, pp. 40-52, 2016.

[45] V. Bruno, G. Battaglia, A. Copani et al., "Metabotropic glutamate receptor subtypes as targets for neuroprotective drugs," Journal of Cerebral Blood Flow \& Metabolism, vol. 21, no. 9, pp. 1013-1033, 2001.

[46] O. Yarishkin, T. T. T. Phuong, C. A. Bretz et al., "TREK-1 channels regulate pressure sensitivity and calcium signaling in trabecular meshwork cells," Journal of General Physiology, vol. 150, no. 12, pp. 1660-1675, 2018.

[47] C. Hivelin, S. Béraud-Dufour, C. Devader et al., "Potentiation of calcium influx and insulin secretion in pancreatic beta cell by the specific TREK-1 blocker spadin," Journal of Diabetes Research, vol. 2016, Article ID 3142175, 9 pages, 2016. 